\title{
New fractional identities, associated novel fractional inequalities with applications to means and error estimations for quadrature formulas
}

\author{
Muhammad Uzair Awan' ${ }^{1}$ Artion Kashuri², Kottakkaran Sooppy Nisar³ , Muhammad Zakria Javed', \\ Sabah Iftikhar ${ }^{4}$, Poom Kumam ${ }^{4,5^{*}}$ (D) and Parin Chaipunya ${ }^{4,6}$ (D)
}

\author{
"Correspondence: \\ poom.kum@kmutt.ac.th \\ ${ }^{4}$ Center of Excellence in Theoretical \\ and Computational Science \\ (TaCS-CoE) \& KMUTT Fixed Point \\ Research Laboratory, Room SCL 802 \\ Fixed Point Laboratory, Science \\ Laboratory Building, Department of \\ Mathematics, King Mongkut's \\ University of Technology Thonburi \\ (KMUTT), 126 Pracha-Uthit Road, \\ Bang Mod, Thung Khru, Bangkok \\ 10140, Thailand \\ ${ }^{5}$ Department of Medical Research, \\ China Medical University Hospital, \\ China Medical University, Taichung \\ 40402, Taiwan \\ Full list of author information is \\ available at the end of the article
}

\begin{abstract}
In this paper, the authors derive some new generalizations of fractional trapezium-like inequalities using the class of harmonic convex functions. Moreover, three new fractional integral identities are given, and on using them as auxiliary results some interesting integral inequalities are found. Finally, in order to show the efficiency of our main results, some applications to special means for different positive real numbers and error estimations for quadrature formulas are obtained.
\end{abstract}

MSC: Primary 26A51; secondary 26A33; 26D07; 26D10; 26D15

Keywords: Trapezium inequality; Harmonic convex function; Riemann-Liouville fractional integrals; Hölder inequality; Power mean inequality; Special means; Error estimations 
Similarly, a function $\Upsilon: S \rightarrow \mathbb{R}$ is said to be convex, if

$$
\Upsilon\left((1-\tau) b_{1}+\tau b_{2}\right) \leq(1-\tau) \Upsilon\left(b_{1}\right)+\tau \Upsilon\left(b_{2}\right), \quad b_{1}, b_{2} \in S, \tau \in[0,1] .
$$

Recently, İșcan [3] introduced the class of harmonic convex functions as:

A function $\Upsilon: I \subset(0,+\infty) \rightarrow \mathbb{R}$ is said to be harmonic convex, if

$$
\Upsilon\left(\frac{b_{1} b_{2}}{\tau b_{1}+(1-\tau) b_{2}}\right) \leq(1-\tau) \Upsilon\left(b_{1}\right)+\tau \Upsilon\left(b_{2}\right), \quad \forall b_{1}, b_{2} \in I, \tau \in[0,1] .
$$

The harmonic property has played a significant role in different fields of pure and applied sciences. In [4] the authors discussed the important role of the harmonic mean in Asian options of stock. Interestingly, harmonic means have applications in electric circuit theory. To be more precise, the total resistance of a set of parallel resistors is just half of the total resistor's harmonic means. For example, if $\mathcal{R}_{1}$ and $\mathcal{R}_{2}$ are the resistances of two parallel resistors, then the total resistance is computed by the formula:

$$
\mathcal{R}_{T}=\frac{\mathcal{R}_{1} \mathcal{R}_{2}}{\mathcal{R}_{1}+\mathcal{R}_{2}}=\frac{1}{2} H\left(\mathcal{R}_{1}, \mathcal{R}_{2}\right)
$$

which is half of the harmonic mean.

Noor [5] showed that the harmonic mean also played a crucial role in developing parallel algorithms for solving nonlinear problems. The author used the harmonic means and harmonic convex functions to suggest some iterative methods for solving linear and nonlinear equations.

The theory of convexity also has a wide range of applications in other areas of pure and applied sciences. It also has a great impact on the development of the theory of inequalities. Several inequalities are consequences of the applications of convex functions. Many generalizations, variants and extensions for the convexity have attracted the attention of many researchers. An interesting result pertaining to convex functions is the trapezium inequality (Hermite-Hadamard inequality) that provides an integral average of a continuous convex function on a compact interval. This result reads as:

Let $\Upsilon: I=\left[b_{1}, b_{2}\right] \subset \mathbb{R} \rightarrow \mathbb{R}$ be a convex function, then

$$
\Upsilon\left(\frac{b_{1}+b_{2}}{2}\right) \leq \frac{1}{b_{2}-b_{1}} \int_{b_{1}}^{b_{2}} \Upsilon(x) \mathrm{d} x \leq \frac{\Upsilon\left(b_{1}\right)+\Upsilon\left(b_{2}\right)}{2} .
$$

Over the years, a variety of new generalizations of this classical result have been obtained in the literature. For example, İșcan [3] obtained a new refinement of the trapezium inequality using the class of harmonic convex functions. He derived the following version of the trapezium inequality.

Let $\Upsilon: I=\left[b_{1}, b_{2}\right] \subset(0,+\infty) \rightarrow \mathbb{R}$ be an harmonic convex function, then

$$
\Upsilon\left(\frac{2 b_{1} b_{2}}{b_{1}+b_{2}}\right) \leq \frac{b_{1} b_{2}}{b_{2}-b_{1}} \int_{b_{1}}^{b_{2}} \frac{\Upsilon(x)}{x^{2}} \mathrm{~d} x \leq \frac{\Upsilon\left(b_{1}\right)+\Upsilon\left(b_{2}\right)}{2} .
$$

We now recall some useful definitions. For brevity, the set of integrable functions on the interval $\left[b_{1}, b_{2}\right]$ is denoted by $L_{1}\left[b_{1}, b_{2}\right]$. 
Definition 1.1 Let $\Upsilon \in L_{1}\left[b_{1}, b_{2}\right]$. The left-and right-sided Riemann-Liouville fractional integrals $J_{b_{1}{ }^{+}}^{\alpha} \Upsilon$ and $J_{b_{2}}^{\alpha} \Upsilon$ of order $\alpha>0$ with $b_{1} \geq 0$ are defined by

$$
J_{b_{1}}^{\alpha} \Upsilon(x)=\frac{1}{\Gamma(\alpha)} \int_{b_{1}}^{x}(x-\tau)^{\alpha-1} \Upsilon(\tau) \mathrm{d} \tau, \quad x>b_{1}
$$

and

$$
J_{b_{2}}^{\alpha} \Upsilon(x)=\frac{1}{\Gamma(\alpha)} \int_{x}^{b_{2}}(\tau-x)^{\alpha-1} \Upsilon(\tau) \mathrm{d} \tau, \quad x<b_{2}
$$

respectively, and $\Gamma(\alpha)$ is Gamma function. Also, we define $J_{b_{1}{ }^{+}}^{0} \Upsilon(x)=J_{b_{2}}^{0} \Upsilon(x)=\Upsilon(x)$.

Definition 1.2 Let $\Upsilon \in L_{1}\left[b_{1}, b_{2}\right]$. The $k$-Riemann-Liouville fractional integrals ${ }_{k} J_{b_{1}}^{\alpha} \Upsilon$ and ${ }_{k} J_{b_{2}}^{\alpha} \Upsilon \Upsilon$ of order $\alpha, k>0$ with $b_{1} \geq 0$ are given as follows:

$$
{ }_{k} J_{b_{1}+}^{\alpha} \Upsilon(x)=\frac{1}{k \Gamma_{k}(\alpha)} \int_{b_{1}}^{x}(x-\tau)^{\frac{\alpha}{k}-1} \Upsilon(\tau) \mathrm{d} \tau, \quad x>b_{1}
$$

and

$$
{ }_{k} J_{b_{2}}^{\alpha} \Upsilon(x)=\frac{1}{k \Gamma_{k}(\alpha)} \int_{x}^{b_{2}}(\tau-x)^{\frac{\alpha}{k}-1} \Upsilon(\tau) \mathrm{d} \tau, \quad x<b_{2},
$$

respectively.

Definition 1.3 A hypergeometric function ${ }_{2} F_{1}\left(b_{1}, b_{2}, b_{3}, z\right)$ has the following integral representation

$$
{ }_{2} F_{1}\left(b_{1}, b_{2}, b_{3}, z\right)=\frac{1}{\beta\left(b_{2}, b_{3}-b_{2}\right)} \int_{0}^{1} x^{b_{2}-1}(1-x)^{b_{3}-b_{2}-1}(1-z x)^{-b_{1}} \mathrm{~d} x, \quad b_{3}>b_{2}>0,
$$

where $\beta(\cdot)$ is the Beta function and $|z|<1$.

Sarikaya et al. [6] opened up a new direction of research in the field of inequalities involving convex functions. They derived a fractional version of the trapezium inequality. This result reads as:

Let $\Upsilon:\left[b_{1}, b_{2}\right] \rightarrow \mathbb{R}$ be a positive function with $0 \leq b_{1}<b_{2}$ and $\Upsilon \in L_{1}\left[b_{1}, b_{2}\right]$. If $\Upsilon$ is a convex function on $\left[b_{1}, b_{2}\right]$, then

$$
\Upsilon\left(\frac{b_{1}+b_{2}}{2}\right) \leq \frac{\Gamma(\alpha+1)}{2\left(b_{2}-b_{1}\right)^{\alpha}}\left[J_{b_{1}}^{\alpha} \Upsilon\left(b_{2}\right)+J_{b_{2}}^{\alpha} \Upsilon\left(b_{1}\right)\right] \leq \frac{\Upsilon\left(b_{1}\right)+\Upsilon\left(b_{2}\right)}{2},
$$

with $\alpha>0$.

Using this idea, İşcan and Wu [7] obtained the fractional trapezium inequality using the class of harmonic convex functions. Their result is stated as follows: 
Let $\Upsilon:\left[b_{1}, b_{2}\right] \subset(0,+\infty) \rightarrow \mathbb{R}$ be a function with $\Upsilon \in L_{1}\left[b_{1}, b_{2}\right]$. If $\Upsilon$ is an harmonic convex function, then

$$
\begin{aligned}
\Upsilon\left(\frac{2 b_{1} b_{2}}{b_{1}+b_{2}}\right) & \leq \frac{\Gamma(\alpha+1)}{2}\left(\frac{b_{1} b_{2}}{b_{2}-b_{1}}\right)^{\alpha}\left[J_{\frac{1}{b_{1}}}^{\alpha} \Upsilon \circ \Psi\left(\frac{1}{b_{2}}\right)+J_{\frac{1}{b_{2}}}^{\alpha} \Upsilon \circ \Psi\left(\frac{1}{b_{1}}\right)\right] \\
& \leq \frac{\Upsilon\left(b_{1}\right)+\Upsilon\left(b_{2}\right)}{2},
\end{aligned}
$$

where $\alpha>0$ and $\Psi(x):=\frac{1}{x}$.

For more details on the trapezium inequality, its generalizations and applications, see $[8,9,17-25,27-31]$.

The aim of this paper is to derive some new generalizations of fractional trapezium-like inequalities using the class of harmonic convex functions. In order to establish some of our main results, we derive three new fractional integral identities. These identities will be used as auxiliary results. Moreover, in order to show the efficiency of our main results, some applications to special means for positive different real numbers and error estimations for quadrature formulas will also be obtained. We also discuss special cases that show that our results represent significant generalizations and under suitable conditions one can obtain many other new and known results.

Before moving to the main results, let us recall some previously known concepts and results that will help us to obtain our main results. Let $\Phi:[0,+\infty) \rightarrow[0,+\infty)$ be a function satisfying the following conditions:

1. $\int_{0}^{1} \frac{\Phi(\tau)}{\tau} \mathrm{d} \tau<+\infty$,

2. $\frac{1}{M_{1}} \leq \frac{\Phi(s)}{\Phi(r)} \leq M_{1}$ for $\frac{1}{2} \leq \frac{s}{r} \leq 2$,

3. $\frac{\Phi(r)}{r^{2}} \leq M_{2} \frac{\Phi(s)}{s^{2}}$ for $s \leq r$,

4. $\left|\frac{\Phi(r)}{r^{2}}-\frac{\Phi(s)}{s^{2}}\right| \leq M_{3}|r-s| \frac{\Phi(r)}{r^{2}}$ for $\frac{1}{2} \leq \frac{s}{r} \leq 2$,

where $M_{1}, M_{2}$ and $M_{3}$ are independent of $r, s>0$. Under the assumptions of $\Phi$, the leftand right-sided generalized fractional integrals are

$$
\begin{aligned}
& { }_{b_{1}}+I_{\Phi} \Upsilon(x)=\int_{b_{1}}^{x} \frac{\Phi(x-\tau)}{x-\tau} \Upsilon(\tau) \mathrm{d} \tau, \quad x>b_{1}, \\
& b_{2}-I_{\Phi} \Upsilon(x)=\int_{x}^{b_{2}} \frac{\Phi(\tau-x)}{\tau-x} \Upsilon(\tau) \mathrm{d} \tau, \quad x<b_{2} .
\end{aligned}
$$

Actually, these fractional integrals are the generalization of some well-known fractional integrals like the Riemann-Liouville fractional integrals [10], the $k$-Riemann-Liouville fractional integrals [11], the Katugampola fractional integrals [12], conformable fractional integrals [13], etc.

1. If we take $\Phi(\tau)=\tau$ in operators (1.1) and (1.2), we have the classical Riemann integrals.

2. If we choose $\Phi(\tau)=\frac{\tau^{\alpha}}{\Gamma(\alpha)}$ in operators (1.1) and (1.2), we obtain the Riemann-Liouville fractional integrals, see [10].

3. If we substitute $\Phi(\tau)=\frac{\tau^{\frac{\alpha}{k}}}{k \Gamma_{k}(\alpha)}$ in operators (1.1) and (1.2), we obtain the $k$-Riemann-Liouville fractional integrals, see [11].

4. If we take $\Phi(\tau)=\tau(x-\tau)^{\alpha-1}$ in operators (1.1) and (1.2), we have conformable fractional integrals that are defined by Khalil et al. [14]. 
5. If we choose $\Phi(\tau)=\frac{\tau}{\alpha} \exp \left(-\frac{1-\alpha}{\alpha} \tau\right)$ for $\alpha \in(0,1]$, in operators (1.1) and (1.2), we get left-sided and right-sided fractional integrals with an exponential kernel that were defined in $[15,16]$.

\section{Main results}

In this section, before we discuss our main results, let us denote, respectively

$$
\begin{aligned}
& \Delta(\tau):=\int_{0}^{\tau} \frac{\Phi\left(\frac{b_{2}-b_{1}}{b_{1} b_{2}} \mu\right)}{\mu} \mathrm{d} \mu \text { and } \delta(\tau):=\int_{\tau}^{1} \frac{\Phi\left(\frac{b_{2}-b_{1}}{b_{1} b_{2}} \mu\right)}{\mu} \mathrm{d} \mu, \\
& \eta(\tau):=\int_{0}^{\tau} \frac{\Phi\left(\frac{b_{2}-b_{1}}{b_{1} b_{2}(m+1)} \mu\right)}{\mu} \mathrm{d} \mu \text { and } \Omega(\tau):=\int_{0}^{\tau} \frac{\Phi\left(\frac{b_{2}-b_{1}}{b_{1} b_{2}(\lambda+\mu)} \mu\right)}{\mu} \mathrm{d} \mu .
\end{aligned}
$$

\subsection{Generalized trapezium inequality}

We now derive a new generalized fractional trapezium-type integral inequality using the class of harmonic convex functions. For brevity, we denote in the following $\Psi(\tau):=\frac{1}{\tau}$.

Theorem 2.1 Let $\Upsilon:\left[b_{1}, b_{2}\right] \rightarrow \mathbb{R}$ be an harmonic convex function, then

$$
\begin{aligned}
\Upsilon\left(\frac{2 b_{1} b_{2}}{b_{1}+b_{2}}\right) & \leq \frac{1}{2 \eta(1)}\left[{ }_{\left(\frac{1}{b_{2}}\right)^{+}} I_{\Phi} \Upsilon \circ \Psi\left(\frac{m b_{1}+b_{2}}{(m+1) b_{1} b_{2}}\right)+{ }_{\left(\frac{1}{b_{1}}\right)^{-}} I_{\Phi} \Upsilon \circ \Psi\left(\frac{b_{1}+m b_{2}}{(m+1) b_{1} b_{2}}\right)\right] \\
& \leq \frac{\left[\Upsilon\left(b_{1}\right)+\Upsilon\left(b_{2}\right)\right]}{2}
\end{aligned}
$$

where $m \in \mathbb{N}$.

Proof Since $\Upsilon$ is an harmonic convex function, then

$$
\Upsilon\left(\frac{2 x y}{x+y}\right) \leq \frac{1}{2}[\Upsilon(x)+\Upsilon(y)]
$$

This implies

$$
2 \Upsilon\left(\frac{2 b_{1} b_{2}}{b_{1}+b_{2}}\right) \leq \Upsilon\left(\frac{(m+1) b_{1} b_{2}}{(1-\tau) b_{1}+(m+\tau) b_{2}}\right)+\Upsilon\left(\frac{(m+1) b_{1} b_{2}}{(m+\tau) b_{1}+(1-\tau) b_{2}}\right) .
$$

Multiplying both sides by $\frac{\Phi\left(\frac{b_{2}-b_{1}}{(m+1) b_{1} b_{2}} \tau\right)}{\tau}$ and integrating with respect to $\tau$ on $[0,1]$, we have

$$
\begin{aligned}
2 \Upsilon( & \left.\frac{2 b_{1} b_{2}}{b_{1}+b_{2}}\right) \int_{0}^{1} \frac{\Phi\left(\frac{b_{2}-b_{1}}{(m+1) b_{1} b_{2}} \tau\right)}{\tau} \mathrm{d} \tau \\
\leq & {\left[\int_{0}^{1} \frac{\Phi\left(\frac{b_{2}-b_{1}}{(m+1) b_{1} b_{2}} \tau\right)}{\tau} \Upsilon\left(\frac{(m+1) b_{1} b_{2}}{(1-\tau) b_{1}+(m+\tau) b_{2}}\right) \mathrm{d} \tau\right.} \\
& \left.+\int_{0}^{1} \frac{\Phi\left(\frac{b_{2}-b_{1}}{(m+1) b_{1} b_{2}} \tau\right)}{\tau} \Upsilon\left(\frac{(m+1) b_{1} b_{2}}{(m+\tau) b_{1}+(1-\tau) b_{2}}\right) \mathrm{d} \tau\right] .
\end{aligned}
$$


This implies

$$
\begin{aligned}
2 \eta(1) \Upsilon\left(\frac{2 b_{1} b_{2}}{b_{1}+b_{2}}\right) \\
\leq \int_{0}^{1} \frac{\Phi\left(\frac{b_{2}-b_{1}}{(m+1) b_{1} b_{2}} \tau\right)}{\tau} \Upsilon\left(\frac{(m+1) b_{1} b_{2}}{(1-\tau) b_{1}+(m+\tau) b_{2}}\right) \mathrm{d} \tau \\
\quad+\int_{0}^{1} \frac{\Phi\left(\frac{b_{2}-b_{1}}{(m+1) b_{1} b_{2}} \tau\right)}{\tau} \Upsilon\left(\frac{(m+1) b_{1} b_{2}}{(m+\tau) b_{1}+(1-\tau) b_{2}}\right) \mathrm{d} \tau \\
=\int_{\frac{1}{b_{2}}}^{\frac{m b_{1}+b_{2}}{(m+1) b_{1} b_{2}}} \frac{\Phi\left(\frac{m b_{1}+b_{2}}{(m+1) b_{1} b_{2}}-x\right)}{\left(\frac{m b_{1}+b_{2}}{(m+1) b_{1} b_{2}}-x\right)} \Upsilon \circ \Psi(x) \mathrm{d} x+\int_{\frac{b_{1}+m b_{2}}{(m+1) b_{1} b_{2}}}^{\frac{1}{b_{1}}} \frac{\left(x-\frac{b_{1}+m b_{2}}{(m+1) b_{1} b_{2}}\right)}{\left(x-\frac{b_{1}+m b_{2}}{(m+1) b_{1} b_{2}}\right)} \Upsilon \Psi(x) \mathrm{d} x \\
={ }_{\left(\frac{1}{b_{2}}\right)^{+}} I_{\Phi} \Upsilon \circ \Psi\left(\frac{m b_{1}+b_{2}}{(m+1) b_{1} b_{2}}\right)+{ }_{\left(\frac{1}{b_{1}}\right)^{-}} I_{\Phi} \Upsilon \circ \Psi\left(\frac{b_{1}+m b_{2}}{(m+1) b_{1} b_{2}}\right) .
\end{aligned}
$$

Now, we prove the second inequality, for this we have

$$
\begin{aligned}
& \Upsilon\left(\frac{(m+1) b_{1} b_{2}}{(1-\tau) b_{1}+(m+\tau) b_{2}}\right) \leq \frac{m+\tau}{m+1} \Upsilon\left(b_{1}\right)+\frac{1-\tau}{m+1} \Upsilon\left(b_{2}\right) . \\
& \Upsilon\left(\frac{(m+1) b_{1} b_{2}}{(m+\tau) b_{1}+(1-\tau) b_{2}}\right) \leq \frac{m+\tau}{m+1} \Upsilon\left(b_{2}\right)+\frac{1-\tau}{m+1} \Upsilon\left(b_{1}\right) .
\end{aligned}
$$

Adding (2.1) and (2.2) and multiplying both sides by $\frac{\Phi\left(\frac{b_{2}-b_{1}}{(m+1) b_{1} b_{2}} \tau\right)}{\tau}$ and integrating with respect to $\tau$ on $[0,1]$, we have

$$
\begin{aligned}
& \int_{0}^{1} \frac{\Phi\left(\frac{b_{2}-b_{1}}{(m+1) b_{1} b_{2}} \tau\right)}{\tau} \Upsilon\left(\frac{(m+1) b_{1} b_{2}}{(1-\tau) b_{1}+(m+\tau) b_{2}}\right) \mathrm{d} \tau \\
& \quad+\int_{0}^{1} \frac{\Phi\left(\frac{b_{2}-b_{1}}{(m+1) b_{1} b_{2}} \tau\right)}{\tau} \Upsilon\left(\frac{(m+1) b_{1} b_{2}}{(m+\tau) b_{1}+(1-\tau) b_{2}}\right) \mathrm{d} \tau \\
& \quad \leq\left[\Upsilon\left(b_{1}\right)+\Upsilon\left(b_{2}\right)\right] \int_{0}^{1} \frac{\Phi\left(\frac{b_{2}-b_{1}}{(m+1) b_{1} b_{2}} \tau\right)}{\tau} \mathrm{d} \tau
\end{aligned}
$$

Using generalized fractional integrals, we obtain our second inequality. This completes the proof.

Corollary 2.1 If we choose $\Phi(\tau)=\tau$ and $m=1$ in Theorem 2.1, we have

$$
\Upsilon\left(\frac{2 b_{1} b_{2}}{b_{1}+b_{2}}\right) \leq \frac{b_{1} b_{2}}{b_{2}-b_{1}} \int_{\frac{1}{b_{2}}}^{\frac{1}{b_{1}}} \Upsilon \circ \Psi(x) \mathrm{d} x \leq \frac{\left[\Upsilon\left(b_{1}\right)+\Upsilon\left(b_{2}\right)\right]}{2} .
$$

Corollary 2.2 If we choose $\Phi(\tau)=\frac{\tau^{\alpha}}{\Gamma(\alpha)}$ in Theorem 2.1, we obtain

$$
\begin{aligned}
\Upsilon\left(\frac{2 b_{1} b_{2}}{b_{1}+b_{2}}\right) \leq & \frac{\left(b_{1} b_{2}(m+1)\right)^{\alpha} \Gamma(\alpha+1)}{2\left(b_{2}-b_{1}\right)^{\alpha}} \\
& \times\left[J_{\left(\frac{1}{b_{2}}\right)^{+}}^{\alpha} \Upsilon \circ \Psi\left(\frac{m b_{1}+b_{2}}{(m+1) b_{1} b_{2}}\right)+J_{\left(\frac{1}{b_{1}}\right)^{-}} \Upsilon \circ \Psi\left(\frac{b_{1}+m b_{2}}{(m+1) b_{1} b_{2}}\right)\right]
\end{aligned}
$$




$$
\leq \frac{\left[\Upsilon\left(b_{1}\right)+\Upsilon\left(b_{2}\right)\right]}{2}
$$

For $m=1$, we obtain

$$
\begin{aligned}
\Upsilon\left(\frac{2 b_{1} b_{2}}{b_{1}+b_{2}}\right) \leq & \frac{2^{\alpha-1}\left(b_{1} b_{2}\right)^{\alpha} \Gamma(\alpha+1)}{\left(b_{2}-b_{1}\right)^{\alpha}} \\
& \times\left[J_{\left(\frac{1}{b_{2}}\right)^{+}}^{\alpha} \Upsilon \circ \Psi\left(\frac{b_{1}+b_{2}}{2 b_{1} b_{2}}\right)+J_{\left(\frac{1}{b_{1}}\right)^{-}} \Upsilon \circ \Psi\left(\frac{b_{1}+b_{2}}{2 b_{1} b_{2}}\right)\right] \\
\leq & \frac{\left[\Upsilon\left(b_{1}\right)+\Upsilon\left(b_{2}\right)\right]}{2} .
\end{aligned}
$$

Corollary 2.3 If we choose $\Phi(\tau)=\frac{\frac{\alpha}{k}}{k \Gamma_{k}(\alpha)}$ in Theorem 2.1, we have

$$
\begin{aligned}
\Upsilon\left(\frac{2 b_{1} b_{2}}{b_{1}+b_{2}}\right) \leq & \frac{\left((m+1) b_{1} b_{2}\right)^{\frac{\alpha}{k}} \Gamma_{k}(\alpha+k)}{2\left(b_{2}-b_{1}\right)^{\frac{\alpha}{k}}} \\
& \times\left[{ }_{k} J_{\left(\frac{1}{b_{2}}\right)^{+}}^{\alpha} \Upsilon \circ \Psi\left(\frac{m b_{1}+b_{2}}{(m+1) b_{1} b_{2}}\right)+{ }_{k} J_{\left(\frac{1}{b_{1}}\right)^{\alpha}}^{\alpha} \Upsilon \circ \Psi\left(\frac{b_{1}+m b_{2}}{(m+1) b_{1} b_{2}}\right)\right] \\
\leq & \frac{\left[\Upsilon\left(b_{1}\right)+\Upsilon\left(b_{2}\right)\right]}{2} .
\end{aligned}
$$

For $m=1$, we obtain

$$
\begin{aligned}
\Upsilon\left(\frac{2 b_{1} b_{2}}{b_{1}+b_{2}}\right) \leq & \frac{2^{\frac{\alpha}{k}-1}\left(b_{1} b_{2}\right)^{\frac{\alpha}{k}} \Gamma_{k}(\alpha+k)}{\left(b_{2}-b_{1}\right)^{\frac{\alpha}{k}}} \\
& \times\left[{ }_{k} J_{\left(\frac{1}{b_{2}}\right)^{+}}^{\alpha} \Upsilon \circ \Psi\left(\frac{b_{1}+b_{2}}{2 b_{1} b_{2}}\right)+{ }_{k} J_{\left(\frac{1}{b_{1}}\right)^{-}}^{\alpha} \Upsilon \circ \Psi\left(\frac{b_{1}+b_{2}}{2 b_{1} b_{2}}\right)\right] \\
\leq & \frac{\left[\Upsilon\left(b_{1}\right)+\Upsilon\left(b_{2}\right)\right]}{2} .
\end{aligned}
$$

\subsection{Auxiliary results}

In this subsection, we derive three new fractional integral identities that will be used in the following.

Lemma 2.2 Let $\Upsilon:\left[b_{1}, b_{2}\right] \rightarrow \mathbb{R}$ be a differentiable function on $\left(b_{1}, b_{2}\right)$ with $b_{1}<b_{2}$ and $m \in \mathbb{N}$, then

$$
\begin{aligned}
& \frac{\Upsilon\left(b_{1}\right)+\Upsilon\left(b_{2}\right)}{m+1} \\
& \quad-\frac{1}{(m+1) \eta(1)}\left[\left(\frac{1}{\left.b_{2}\right)}{ }^{+} I_{\Phi} \Upsilon \circ \Psi\left(\frac{m b_{1}+b_{2}}{(m+1) b_{1} b_{2}}\right)+{ }_{\left(\frac{1}{b_{1}}\right)-I_{\Phi}} \Upsilon \circ \Psi\left(\frac{b_{1}+m b_{2}}{(m+1) b_{1} b_{2}}\right)\right]\right. \\
& \quad=\frac{b_{1} b_{2}\left(b_{2}-b_{1}\right)}{\eta(1)}\left[\int_{0}^{1} \frac{\eta(\tau)}{\left((m+\tau) b_{1}+(1-\tau) b_{2}\right)^{2}} \Upsilon^{\prime}\left(\frac{(m+1) b_{1} b_{2}}{(m+\tau) b_{1}+(1-\tau) b_{2}}\right) \mathrm{d} \tau\right. \\
& \left.\quad-\int_{0}^{1} \frac{\eta(\tau)}{\left((1-\tau) b_{1}+(m+\tau) b_{2}\right)^{2}} \Upsilon^{\prime}\left(\frac{(m+1) b_{1} b_{2}}{(1-\tau) b_{1}+(m+\tau) b_{2}}\right) \mathrm{d} \tau\right] .
\end{aligned}
$$


Proof Consider the right-hand side

$$
\begin{aligned}
I:= & \frac{b_{1} b_{2}\left(b_{2}-b_{1}\right)}{\eta(1)}\left[\int_{0}^{1} \frac{\eta(\tau)}{\left((m+\tau) b_{1}+(1-\tau) b_{2}\right)^{2}} \Upsilon^{\prime}\left(\frac{(m+1) b_{1} b_{2}}{(m+\tau) b_{1}+(1-\tau) b_{2}}\right) \mathrm{d} \tau\right. \\
& \left.-\int_{0}^{1} \frac{\eta(\tau)}{\left((1-\tau) b_{1}+(m+\tau) b_{2}\right)^{2}} \Upsilon^{\prime}\left(\frac{(m+1) b_{1} b_{2}}{(1-\tau) b_{1}+(m+\tau) b_{2}}\right) \mathrm{d} \tau\right] \\
= & \frac{b_{1} b_{2}\left(b_{2}-b_{1}\right)}{\eta(1)}\left[I_{1}-I_{2}\right],
\end{aligned}
$$

where

$$
\begin{aligned}
I_{1}:= & \int_{0}^{1} \frac{\eta(\tau)}{\left((m+\tau) b_{1}+(1-\tau) b_{2}\right)^{2}} \Upsilon^{\prime}\left(\frac{(m+1) b_{1} b_{2}}{(m+\tau) b_{1}+(1-\tau) b_{2}}\right) \mathrm{d} \tau \\
= & \frac{\eta(1) \Upsilon\left(b_{2}\right)}{(m+1) b_{1} b_{2}\left(b_{2}-b_{1}\right)} \\
& -\frac{1}{b_{1} b_{2}\left(b_{2}-b_{1}\right)(m+1)} \int_{\frac{1}{b_{2}}}^{\frac{m b_{1}+b_{2}}{(m+1) b_{1} b_{2}}} \frac{\Phi\left(\frac{m b_{1}+b_{2}}{(m+1) b_{1} b_{2}}-x\right)}{\frac{m b_{1}+b_{2}}{(m+1) b_{1} b_{2}}-x} \Upsilon \circ \Psi(x) \mathrm{d} x \\
= & \frac{\eta(1) \Upsilon\left(b_{2}\right)}{(m+1) b_{1} b_{2}\left(b_{2}-b_{1}\right)}-\frac{1}{b_{1} b_{2}\left(b_{2}-b_{1}\right)(m+1)}\left(\frac{1}{b_{2}}\right)^{+} I_{\Phi} \Upsilon \circ \Psi\left(\frac{m b_{1}+b_{2}}{(m+1) b_{1} b_{2}}\right) .
\end{aligned}
$$

Similarly,

$$
\begin{aligned}
I_{2}:= & \int_{0}^{1} \frac{\eta(\tau)}{\left((1-\tau) b_{1}+(m+\tau) b_{2}\right)^{2}} \Upsilon^{\prime}\left(\frac{(m+1) b_{1} b_{2}}{(1-\tau) b_{1}+(m+\tau) b_{2}}\right) \mathrm{d} \tau \\
= & -\frac{\eta(1) \Upsilon\left(b_{1}\right)}{(m+1) b_{1} b_{2}\left(b_{2}-b_{1}\right)} \\
& +\frac{1}{b_{1} b_{2}\left(b_{2}-b_{1}\right)(m+1)} \int_{\frac{b_{1}+m b_{2}}{(m+1) b_{1} b_{2}}}^{\frac{1}{b_{1}}} \frac{\Phi\left(x-\frac{b_{1}+m b_{2}}{(m+1) b_{1} b_{2}}\right)}{x-\frac{b_{1}+m b_{2}}{(m+1) b_{1} b_{2}}} \Upsilon \odot \Psi(x) \mathrm{d} x \\
= & -\frac{\eta(1) \Upsilon\left(b_{1}\right)}{(m+1) b_{1} b_{2}\left(b_{2}-b_{1}\right)}+\frac{1}{b_{1} b_{2}\left(b_{2}-b_{1}\right)(m+1)}\left(\frac{1}{b_{1}}\right)^{-} I_{\Phi} \Upsilon \circ \Psi\left(\frac{b_{1}+m b_{2}}{(m+1) b_{1} b_{2}}\right) .
\end{aligned}
$$

Substituting the values of $I_{1}$ and $I_{2}$ in $I$, we obtain our required result.

Remark 2.1 If we choose $m=1$ and $\Phi(\tau)=\tau$, we have

$$
\begin{aligned}
& \frac{\Upsilon\left(b_{1}\right)+\Upsilon\left(b_{2}\right)}{2}-\frac{b_{1} b_{2}}{\left(b_{2}-b_{1}\right)} \int_{\frac{1}{b_{2}}}^{\frac{1}{b_{1}}} \Upsilon \circ \Psi(x) \mathrm{d} x \\
& \quad=b_{1} b_{2}\left(b_{2}-b_{1}\right)\left[\int_{0}^{1} \frac{\tau}{\left((1+\tau) b_{1}+(1-\tau) b_{2}\right)^{2}} \Upsilon^{\prime}\left(\frac{2 b_{1} b_{2}}{(1+\tau) b_{1}+(1-\tau) b_{2}}\right) \mathrm{d} \tau\right. \\
& \left.\quad-\int_{0}^{1} \frac{\tau}{\left((1-\tau) b_{1}+(1+\tau) b_{2}\right)^{2}} \Upsilon^{\prime}\left(\frac{2 b_{1} b_{2}}{(1-\tau) b_{1}+(1+\tau) b_{2}}\right) \mathrm{d} \tau\right] .
\end{aligned}
$$


Corollary 2.4 If we take $m=1$ and $\Phi(\tau)=\frac{\tau^{\alpha}}{\Gamma(\alpha)}$ in Lemma 2.2, we obtain

$$
\begin{aligned}
& \frac{\Upsilon\left(b_{1}\right)+\Upsilon\left(b_{2}\right)}{2} \\
& \quad-\frac{2^{\alpha-1}\left(b_{1} b_{2}\right)^{\alpha} \Gamma(\alpha+1)}{\left(b_{2}-b_{1}\right)^{\alpha}}\left[J_{\left(\frac{1}{b_{2}}\right)^{+}}^{\alpha} \Upsilon \Psi\left(\frac{b_{1}+b_{2}}{2 b_{1} b_{2}}\right)+J_{\left(\frac{1}{b_{1}}\right)^{-}}^{\alpha} \Upsilon \circ \Psi\left(\frac{b_{1}+b_{2}}{2 b_{1} b_{2}}\right)\right] \\
& \quad b_{1} b_{2}\left(b_{2}-b_{1}\right)\left[\int_{0}^{1} \frac{\tau^{\alpha}}{\left((1+\tau) b_{1}+(1-\tau) b_{2}\right)^{2}} \Upsilon^{\prime}\left(\frac{2 b_{2}}{(1+\tau) b_{1}+(1-\tau) b_{2}}\right) \mathrm{d} \tau\right. \\
& \left.\quad-\int_{0}^{1} \frac{2 b_{1} b_{2}}{\left((1-\tau) b_{1}+(1+\tau) b_{2}\right)^{2}} \Upsilon^{\prime}\left(\frac{\tau^{\alpha}}{(1-\tau) b_{1}+(1-\tau) b_{2}}\right) \mathrm{d} \tau\right]
\end{aligned}
$$

Corollary 2.5 If we choose $m=1$ and $\Phi(\tau)=\frac{\tau^{\frac{\alpha}{k}}}{k \Gamma_{k}(\alpha)}$ in Lemma 2.2, we obtain

$$
\begin{aligned}
& \frac{\Upsilon\left(b_{1}\right)+\Upsilon\left(b_{2}\right)}{2} \\
& \quad-\frac{2^{\frac{\alpha}{k}-1}\left(b_{1} b_{2}\right)^{\frac{\alpha}{k}} \Gamma_{k}(\alpha+k)}{\left(b_{2}-b_{1}\right)^{\frac{\alpha}{k}}}\left[{ }_{k} J_{\left(\frac{1}{b_{2}}\right)^{+}}^{\alpha} \Upsilon \Psi\left(\frac{b_{1}+b_{2}}{2 b_{1} b_{2}}\right)+{ }_{k} J_{\left(\frac{1}{b_{1}}\right)^{-}}^{\alpha} \Upsilon \circ \Psi\left(\frac{b_{1}+b_{2}}{2 b_{1} b_{2}}\right)\right] \\
& =b_{1} b_{2}\left(b_{2}-b_{1}\right)\left[\int_{0}^{1} \frac{2 b_{1} b_{2}}{\left((1+\tau) b_{1}+(1-\tau) b_{2}\right)^{2}} \Upsilon^{\prime}\left(\frac{\tau^{\frac{\alpha}{k}}}{(1+\tau) b_{1}+(1-\tau) b_{2}}\right) \mathrm{d} \tau\right. \\
& \left.\quad-\int_{0}^{1} \frac{2 b_{1} b_{2}}{\left((1-\tau) b_{1}+(1+\tau) b_{2}\right)^{2}} \Upsilon^{\prime}\left(\frac{\alpha}{k}\right) \mathrm{d} \tau\right] .
\end{aligned}
$$

Lemma 2.3 Let $\Upsilon:\left[b_{1}, b_{2}\right] \rightarrow \mathbb{R}$ be a differentiable function on $\left(b_{1}, b_{2}\right)$ with $b_{1}<b_{2}$ and $\lambda, \mu \in[0, \infty)$ with $\lambda+\mu \neq 0$, then

$$
\begin{aligned}
& \frac{\Omega(\lambda) \Upsilon\left(b_{2}\right)+\Omega(\mu) \Upsilon\left(b_{1}\right)}{\lambda+\mu} \\
& -\frac{1}{\lambda+\mu}\left[\left(\frac{1}{b_{2}}{ }^{+} I_{\Phi} \Upsilon \circ \Psi\left(\frac{\lambda b_{2}+\mu b_{1}}{b_{1} b_{2}(\lambda+\mu)}\right)+{ }_{\left(\frac{1}{b_{1}}\right)^{-}} I_{\Phi} \Upsilon \circ \Psi\left(\frac{\lambda b_{2}+\mu b_{1}}{b_{1} b_{2}(\lambda+\mu)}\right)\right]\right. \\
& =b_{1} b_{2}\left(b_{2}-b_{1}\right)\left[\int_{0}^{\lambda} \frac{\Omega(\tau)}{\left((\lambda-\tau) b_{2}+(\mu+\tau) b_{1}\right)^{2}} \Upsilon^{\prime}\left(\frac{b_{1} b_{2}(\lambda+\mu)}{(\lambda-\tau) b_{2}+(\mu+\tau) b_{1}}\right) \mathrm{d} \tau\right. \\
& \left.-\int_{0}^{\mu} \frac{\Omega(\tau)}{\left((\lambda+\tau) b_{2}+(\mu-\tau) b_{1}\right)^{2}} \Upsilon^{\prime}\left(\frac{b_{1} b_{2}(\lambda+\mu)}{(\lambda+\tau) b_{2}+(\mu-\tau) b_{1}}\right) \mathrm{d} \tau\right] .
\end{aligned}
$$

Proof Consider the right-hand side

$$
\begin{aligned}
I:= & b_{1} b_{2}\left(b_{2}-b_{1}\right)\left[\int_{0}^{\lambda} \frac{\Omega(\tau)}{\left((\lambda-\tau) b_{2}+(\mu+\tau) b_{1}\right)^{2}} \Upsilon^{\prime}\left(\frac{b_{1} b_{2}(\lambda+\mu)}{(\lambda-\tau) b_{2}+(\mu+\tau) b_{1}}\right) \mathrm{d} \tau\right. \\
& \left.-\int_{0}^{\mu} \frac{\Omega(\tau)}{\left((\lambda+\tau) b_{2}+(\mu-\tau) b_{1}\right)^{2}} \Upsilon^{\prime}\left(\frac{b_{1} b_{2}(\lambda+\mu)}{(\lambda+\tau) b_{2}+(\mu-\tau) b_{1}}\right) \mathrm{d} \tau\right] \\
= & b_{1} b_{2}\left(b_{2}-b_{1}\right)\left[I_{3}-I_{4}\right],
\end{aligned}
$$


where

$$
\begin{aligned}
I_{3}:= & \int_{0}^{\lambda} \frac{\Omega(\tau)}{\left((\lambda-\tau) b_{2}+(\mu+\tau) b_{1}\right)^{2}} \Upsilon^{\prime}\left(\frac{b_{1} b_{2}(\lambda+\mu)}{(\lambda-\tau) b_{2}+(\mu+\tau) b_{1}}\right) \mathrm{d} \tau \\
= & \frac{\Omega(\lambda) \Upsilon\left(b_{2}\right)}{b_{1} b_{2}\left(b_{2}-b_{1}\right)(\lambda+\mu)} \\
& -\frac{1}{b_{1} b_{2}\left(b_{2}-b_{1}\right)(\lambda+\mu)} \int_{\frac{1}{b_{2}}}^{\frac{\lambda b_{2}+\mu b_{1}}{b_{1} b_{2}(\lambda+\mu)}} \frac{\Phi\left(\frac{\lambda b_{2}+\mu b_{1}}{b_{1} b_{2}(\lambda+\mu)}-x\right)}{\left(\frac{\lambda b_{2}+\mu b_{1}}{b_{1} b_{2}(\lambda+\mu)}-x\right)} \Upsilon \circ \Psi(x) \mathrm{d} x \\
= & \frac{\Omega(\lambda) \Upsilon\left(b_{2}\right)}{b_{1} b_{2}\left(b_{2}-b_{1}\right)(\lambda+\mu)}-\frac{1}{b_{1} b_{2}\left(b_{2}-b_{1}\right)(\lambda+\mu)^{(}\left(\frac{1}{b_{2}}\right)+I_{\Phi} \Upsilon \circ \Psi\left(\frac{\lambda b_{2}+\mu b_{1}}{b_{1} b_{2}(\lambda+\mu)}\right) .} .
\end{aligned}
$$

Similarly,

$$
\begin{aligned}
I_{4}:= & \int_{0}^{\mu} \frac{\Omega(\tau)}{\left((\lambda+\tau) b_{2}+(\mu-\tau) b_{1}\right)^{2}} \Upsilon^{\prime}\left(\frac{b_{1} b_{2}(\lambda+\mu)}{(\lambda+\tau) b_{2}+(\mu-\tau) b_{1}}\right) \mathrm{d} \tau \\
= & -\frac{\Omega(\mu) \Upsilon\left(b_{1}\right)}{b_{1} b_{2}\left(b_{2}-b_{1}\right)(\lambda+\mu)} \\
& +\frac{1}{b_{1} b_{2}\left(b_{2}-b_{1}\right)(\lambda+\mu)} \int_{\frac{\lambda b_{2}+\mu b_{1}}{b_{1} b_{2}(\lambda+\mu)}}^{\frac{1}{b_{1}}} \frac{\Phi\left(x-\frac{\lambda b_{2}+\mu b_{1}}{b_{1} b_{2}(\lambda+\mu)}\right)}{\left(x-\frac{\lambda b_{2}+\mu b_{1}}{b_{1} b_{2}(\lambda+\mu)}\right)} \Upsilon \circ \Psi(x) \mathrm{d} x \\
= & -\frac{\Omega(\mu) \Upsilon\left(b_{2}\right)}{b_{1} b_{2}\left(b_{2}-b_{1}\right)(\lambda+\mu)}-\frac{1}{b_{1} b_{2}\left(b_{2}-b_{1}\right)(\lambda+\mu)^{(}}\left(^{\frac{1}{b_{1}}}\right)^{-} I_{\Phi} \Upsilon \circ \Psi\left(\frac{\lambda b_{2}+\mu b_{1}}{b_{1} b_{2}(\lambda+\mu)}\right) .
\end{aligned}
$$

Substituting the values of $I_{3}$ and $I_{4}$ in (2.3), we obtain our required result.

Corollary 2.6 Choosing $\Phi(\tau)=\tau$ in Lemma 2.3, we have

$$
\begin{aligned}
& \frac{\lambda \Upsilon\left(b_{2}\right)+\mu \Upsilon\left(b_{1}\right)}{\lambda+\mu}-\frac{b_{1} b_{2}}{b_{2}-b_{1}} \int_{\frac{1}{b_{2}}}^{\frac{1}{b_{1}}} \Upsilon \circ \Psi(x) \mathrm{d} x \\
& =b_{1} b_{2}\left(b_{2}-b_{1}\right)\left[\int_{0}^{\lambda} \frac{\tau}{\left((\lambda-\tau) b_{2}+(\mu+\tau) b_{1}\right)^{2}} \Upsilon^{\prime}\left(\frac{b_{1} b_{2}(\lambda+\mu)}{(\lambda-\tau) b_{2}+(\mu+\tau) b_{1}}\right) \mathrm{d} \tau\right. \\
& \left.\quad-\int_{0}^{\mu} \frac{\tau}{\left((\lambda+\tau) b_{2}+(\mu-\tau) b_{1}\right)^{2}} \Upsilon^{\prime}\left(\frac{b_{1} b_{2}(\lambda+\mu)}{(\lambda+\tau) b_{2}+(\mu-\tau) b_{1}}\right) \mathrm{d} \tau\right] .
\end{aligned}
$$

Corollary 2.7 Taking $\Phi(\tau)=\frac{\tau^{\alpha}}{\Gamma(\alpha)}$ in Lemma 2.3, we obtain

$$
\begin{aligned}
& \frac{\lambda^{\alpha} \Upsilon\left(b_{2}\right)+\mu^{\alpha} \Upsilon\left(b_{1}\right)}{\lambda+\mu}-\frac{\left(b_{1} b_{2}\right)^{\alpha}(\lambda+\mu)^{\alpha-1} \Gamma(\alpha+1)}{\left(b_{2}-b_{1}\right)^{\alpha}}\left[J_{\left(\frac{1}{b_{2}}\right)^{+}}^{\alpha} \Upsilon \circ \Psi\left(\frac{\lambda b_{2}+\mu b_{1}}{b_{1} b_{2}(\lambda+\mu)}\right)\right. \\
& \left.\quad+J_{\left(\frac{1}{b_{1}}\right)^{-}}^{\alpha} \Upsilon \circ \Psi\left(\frac{\lambda b_{2}+\mu b_{1}}{b_{1} b_{2}(\lambda+\mu)}\right)\right] \\
& =b_{1} b_{2}\left(b_{2}-b_{1}\right)\left[\int_{0}^{\lambda} \frac{\tau^{\alpha}}{\left((\lambda-\tau) b_{2}+(\mu+\tau) b_{1}\right)^{2}} \Upsilon^{\prime}\left(\frac{b_{1} b_{2}(\lambda+\mu)}{(\lambda-\tau) b_{2}+(\mu+\tau) b_{1}}\right) \mathrm{d} \tau\right. \\
& \left.\quad-\int_{0}^{\mu} \frac{\tau^{\alpha}}{\left((\lambda+\tau) b_{2}+(\mu-\tau) b_{1}\right)^{2}} \Upsilon^{\prime}\left(\frac{b_{1} b_{2}(\lambda+\mu)}{(\lambda+\tau) b_{2}+(\mu-\tau) b_{1}}\right) \mathrm{d} \tau\right] .
\end{aligned}
$$


Corollary 2.8 Choosing $\Phi(\tau)=\frac{\tau^{\frac{\alpha}{k}}}{k \Gamma_{k}(\alpha)}$ in Lemma 2.3, we obtain

$$
\begin{aligned}
& \frac{\lambda^{\frac{\alpha}{k}} \Upsilon\left(b_{2}\right)+\mu^{\frac{\alpha}{k}} \Upsilon\left(b_{1}\right)}{\lambda+\mu}-\frac{k\left(b_{1} b_{2}\right)^{\frac{\alpha}{k}}(\lambda+\mu)^{\frac{\alpha}{k}-1} \Gamma_{k}(\alpha+k)}{\left(b_{2}-b_{1}\right)^{\frac{\alpha}{k}}}\left[{ }_{k} J_{\left(\frac{1}{b_{2}}\right)^{+}}^{\alpha} \Upsilon \circ \Psi\left(\frac{\lambda b_{2}+\mu b_{1}}{b_{1} b_{2}(\lambda+\mu)}\right)\right. \\
& \left.\quad+{ }_{k} J_{\left(\frac{1}{b_{1}}\right)^{-}}^{\alpha} \Upsilon \Psi\left(\frac{\lambda b_{2}+\mu b_{1}}{b_{1} b_{2}(\lambda+\mu)}\right)\right] \\
& =b_{1} b_{2}\left(b_{2}-b_{1}\right)\left[\int_{0}^{\lambda} \frac{\tau^{\frac{\alpha}{k}}}{\left((\lambda-\tau) b_{2}+(\mu+\tau) b_{1}\right)^{2}} \Upsilon^{\prime}\left(\frac{b_{1} b_{2}(\lambda+\mu)}{(\lambda-\tau) b_{2}+(\mu+\tau) b_{1}}\right) \mathrm{d} \tau\right. \\
& \left.\quad-\int_{0}^{\mu} \frac{\tau^{\frac{\alpha}{k}}}{\left((\lambda+\tau) b_{2}+(\mu-\tau) b_{1}\right)^{2}} \Upsilon^{\prime}\left(\frac{b_{1} b_{2}(\lambda+\mu)}{(\lambda+\tau) b_{2}+(\mu-\tau) b_{1}}\right) \mathrm{d} \tau\right] .
\end{aligned}
$$

Lemma 2.4 Let $\Upsilon:\left[b_{1}, b_{2}\right] \subset(0,+\infty) \rightarrow \mathbb{R}$ be a differentiable mapping on $\left(b_{1}, b_{2}\right)$ with $b_{1}<b_{2}$, then

$$
\begin{aligned}
& \Upsilon\left(\frac{2 b_{1} b_{2}}{b_{1}+b_{2}}\right)-\frac{1}{2 \Delta(1)}\left[{\frac{1}{b_{2}}}^{+} I_{\Phi} \Upsilon \circ \Psi\left(\frac{1}{b_{1}}\right)+{\frac{1}{b_{1}}}-I_{\Phi} \Upsilon \circ \Psi\left(\frac{1}{b_{2}}\right)\right] \\
& \quad=\frac{b_{1} b_{2}\left(b_{2}-b_{1}\right)}{2 \Delta(1)} \sum_{j=1}^{4} M_{j},
\end{aligned}
$$

where

$$
\begin{aligned}
& M_{1}:=\int_{0}^{\frac{1}{2}} \frac{\Delta(\tau)}{\left(\tau b_{1}+(1-\tau) b_{2}\right)^{2}} \Upsilon^{\prime}\left(\frac{b_{1} b_{2}}{\tau b_{1}+(1-\tau) b_{2}}\right) \mathrm{d} \tau, \\
& M_{2}:=\int_{0}^{\frac{1}{2}} \frac{(-\Delta(\tau))}{\left((1-\tau) b_{1}+\tau b_{2}\right)^{2}} \Upsilon^{\prime}\left(\frac{b_{1} b_{2}}{(1-\tau) b_{1}+\tau b_{2}}\right) \mathrm{d} \tau, \\
& M_{3}:=\int_{\frac{1}{2}}^{1} \frac{(-\delta(\tau))}{\left(\tau b_{1}+(1-\tau) b_{2}\right)^{2}} \Upsilon^{\prime}\left(\frac{b_{1} b_{2}}{\tau b_{1}+(1-\tau) b_{2}}\right) \mathrm{d} \tau, \\
& M_{4}:=\int_{\frac{1}{2}}^{1} \frac{\delta(\tau)}{\left((1-\tau) b_{1}+\tau b_{2}\right)^{2}} \Upsilon^{\prime}\left(\frac{b_{1} b_{2}}{(1-\tau) b_{1}+\tau b_{2}}\right) \mathrm{d} \tau .
\end{aligned}
$$

Proof Integrating by parts $M_{i}$ for $i=1,2,3,4$, and changing the variables, we have

$$
\begin{aligned}
M_{1}= & \frac{1}{b_{1} b_{2}\left(b_{2}-b_{1}\right)} \Upsilon\left(\frac{2 b_{1} b_{2}}{b_{1}+b_{2}}\right) \int_{0}^{\frac{1}{2}} \frac{\Phi\left(\frac{b_{2}-b_{1}}{b_{1} b_{2}} \mu\right)}{\mu} \mathrm{d} \mu \\
& -\frac{1}{b_{1} b_{2}\left(b_{2}-b_{1}\right)} \int_{0}^{\frac{1}{2}} \frac{\Phi\left(\frac{b_{2}-b_{1}}{b_{1} b_{2}} \tau\right)}{\tau} \Upsilon\left(\frac{b_{1} b_{2}}{\tau b_{1}+(1-\tau) b_{2}}\right) \mathrm{d} \tau, \\
M_{2}= & \frac{1}{b_{1} b_{2}\left(b_{2}-b_{1}\right)} \Upsilon\left(\frac{2 b_{1} b_{2}}{b_{1}+b_{2}}\right) \int_{0}^{\frac{1}{2}} \frac{\Phi\left(\frac{b_{2}-b_{1}}{b_{1} b_{2}} \mu\right)}{\mu} \mathrm{d} \mu \\
& -\frac{1}{b_{1} b_{2}\left(b_{2}-b_{1}\right)} \int_{0}^{\frac{1}{2}} \frac{\Phi\left(\frac{b_{2}-b_{1}}{b_{1} b_{2}} \tau\right)}{\tau} \Upsilon\left(\frac{b_{1} b_{2}}{(1-\tau) b_{1}+\tau b_{2}}\right) \mathrm{d} \tau,
\end{aligned}
$$




$$
\begin{aligned}
M_{3}= & \frac{1}{b_{1} b_{2}\left(b_{2}-b_{1}\right)} \Upsilon\left(\frac{2 b_{1} b_{2}}{b_{1}+b_{2}}\right) \int_{\frac{1}{2}}^{1} \frac{\Phi\left(\frac{b_{2}-b_{1}}{b_{1} b_{2}} \mu\right)}{\mu} \mathrm{d} \mu \\
& -\frac{1}{b_{1} b_{2}\left(b_{2}-b_{1}\right)} \int_{\frac{1}{2}}^{1} \frac{\Phi\left(\frac{b_{2}-b_{1}}{b_{1} b_{2}} \tau\right)}{\tau} \Upsilon\left(\frac{b_{1} b_{2}}{\tau b_{1}+(1-\tau) b_{2}}\right) \mathrm{d} \tau, \\
M_{4}= & \frac{1}{b_{1} b_{2}\left(b_{2}-b_{1}\right)} \Upsilon\left(\frac{2 b_{1} b_{2}}{b_{1}+b_{2}}\right) \int_{\frac{1}{2}}^{1} \frac{\Phi\left(\frac{b_{2}-b_{1}}{b_{1} b_{2}} \mu\right)}{\mu} \mathrm{d} \mu \\
& -\frac{1}{b_{1} b_{2}\left(b_{2}-b_{1}\right)} \int_{\frac{1}{2}}^{1} \frac{\Phi\left(\frac{b_{2}-b_{1}}{b_{1} b_{2}} \tau\right)}{\tau} \Upsilon\left(\frac{b_{1} b_{2}}{(1-\tau) b_{1}+\tau b_{2}}\right) \mathrm{d} \tau .
\end{aligned}
$$

Adding $M_{1}, M_{2}, M_{3}$ and $M_{4}$ and multiplying by the factor $\frac{b_{1} b_{2}\left(b_{2}-b_{1}\right)}{2 \Delta(1)}$, we obtain our required result.

Corollary 2.9 Taking $\Phi(\tau)=\tau$ in Lemma 2.4, then

$$
\Upsilon\left(\frac{2 b_{1} b_{2}}{b_{1}+b_{2}}\right)-\frac{b_{1} b_{2}}{b_{2}-b_{1}} \int_{b_{1}}^{b_{2}} \frac{\Upsilon(x)}{x^{2}} \mathrm{~d} x=\frac{b_{1} b_{2}\left(b_{2}-b_{1}\right)}{2} \sum_{j=1}^{4} L_{j},
$$

where

$$
\begin{aligned}
& L_{1}:=\int_{0}^{\frac{1}{2}} \frac{\tau}{\left(\tau b_{1}+(1-\tau) b_{2}\right)^{2}} \Upsilon^{\prime}\left(\frac{b_{1} b_{2}}{\tau b_{1}+(1-\tau) b_{2}}\right) \mathrm{d} \tau, \\
& L_{2}:=\int_{0}^{\frac{1}{2}} \frac{-\tau}{\left((1-\tau) b_{1}+\tau b_{2}\right)^{2}} \Upsilon^{\prime}\left(\frac{b_{1} b_{2}}{\tau b_{1}+(1-\tau) b_{2}}\right) \mathrm{d} \tau, \\
& L_{3}:=\int_{\frac{1}{2}}^{1} \frac{-\tau}{\left(\tau b_{1}+(1-\tau) b_{2}\right)^{2}} \Upsilon^{\prime}\left(\frac{b_{1} b_{2}}{\tau b_{1}+(1-\tau) b_{2}}\right) \mathrm{d} \tau, \\
& L_{4}:=\int_{\frac{1}{2}}^{1} \frac{\tau}{\left((1-\tau) b_{1}+\tau b_{2}\right)^{2}} \Upsilon^{\prime}\left(\frac{b_{1} b_{2}}{(1-\tau) b_{1}+\tau b_{2}}\right) \mathrm{d} \tau .
\end{aligned}
$$

Corollary 2.10 Choosing $\Phi(\tau)=\frac{\tau^{\alpha}}{\Gamma(\alpha)}$ in Lemma 2.4, then

$$
\begin{aligned}
& \Upsilon\left(\frac{2 b_{1} b_{2}}{b_{1}+b_{2}}\right)-\frac{\Gamma(\alpha+1)}{2}\left(\frac{b_{1} b_{2}}{b_{2}-b_{1}}\right)^{\alpha}\left[J_{\frac{1}{b_{2}}}^{\alpha} \Upsilon \circ \Psi\left(\frac{1}{b_{1}}\right)+J_{\frac{1}{b_{1}}}^{\alpha} \Upsilon \circ \Psi\left(\frac{1}{b_{2}}\right)\right] \\
& \quad=\frac{b_{1} b_{2}\left(b_{2}-b_{1}\right)}{2} \sum_{j=5}^{8} L_{j},
\end{aligned}
$$

where

$$
\begin{aligned}
& L_{5}:=\int_{0}^{\frac{1}{2}} \frac{\tau^{\alpha}}{\left(\tau b_{1}+(1-\tau) b_{2}\right)^{2}} \Upsilon^{\prime}\left(\frac{b_{1} b_{2}}{\tau b_{1}+(1-\tau) b_{2}}\right) \mathrm{d} \tau, \\
& L_{6}:=\int_{0}^{\frac{1}{2}} \frac{(-\tau)^{\alpha}}{\left((1-\tau) b_{1}+\tau b_{2}\right)^{2}} \Upsilon^{\prime}\left(\frac{b_{1} b_{2}}{(1-\tau) b_{1}+\tau b_{2}}\right) \mathrm{d} \tau, \\
& L_{7}:=\int_{\frac{1}{2}}^{1} \frac{(-\tau)^{\alpha}}{\left(\tau b_{1}+(1-\tau) b_{2}\right)^{2}} \Upsilon^{\prime}\left(\frac{b_{1} b_{2}}{\tau b_{1}+(1-\tau) b_{2}}\right) \mathrm{d} \tau,
\end{aligned}
$$




$$
L_{8}:=\int_{\frac{1}{2}}^{1} \frac{\tau^{\alpha}}{\left((1-\tau) b_{1}+\tau b_{2}\right)^{2}} \Upsilon^{\prime}\left(\frac{b_{1} b_{2}}{(1-\tau) b_{1}+\tau b_{2}}\right) \mathrm{d} \tau
$$

Corollary 2.11 Taking $\Phi(\tau)=\frac{\tau^{\frac{\alpha}{k}}}{k \Gamma_{k}(\alpha)}$ in Lemma 2.4, then

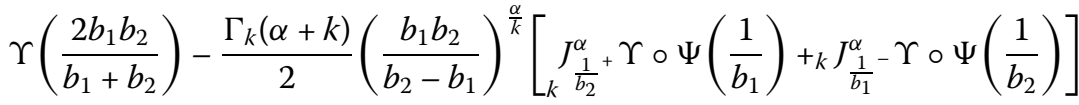

$$
\begin{aligned}
& =\frac{b_{1} b_{2}\left(b_{2}-b_{1}\right)}{2} \sum_{j=9}^{12} L_{j}
\end{aligned}
$$

where

$$
\begin{aligned}
& L_{9}:=\int_{0}^{\frac{1}{2}} \frac{\tau^{\frac{\alpha}{k}}}{\left(\tau b_{1}+(1-\tau) b_{2}\right)^{2}} \Upsilon^{\prime}\left(\frac{b_{1} b_{2}}{\tau b_{1}+(1-\tau) b_{2}}\right) \mathrm{d} \tau, \\
& L_{10}:=\int_{0}^{\frac{1}{2}} \frac{(-\tau)^{\frac{\alpha}{k}}}{\left((1-\tau) b_{1}+\tau b_{2}\right)^{2}} \Upsilon^{\prime}\left(\frac{b_{1} b_{2}}{(1-\tau) b_{1}+\tau b_{2}}\right) \mathrm{d} \tau, \\
& L_{11}:=\int_{\frac{1}{2}}^{1} \frac{(-\tau)^{\frac{\alpha}{k}}}{\left(\tau b_{1}+(1-\tau) b_{2}\right)^{2}} \Upsilon^{\prime}\left(\frac{b_{1} b_{2}}{\tau b_{1}+(1-\tau) b_{2}}\right) \mathrm{d} \tau, \\
& L_{12}:=\int_{\frac{1}{2}}^{1} \frac{\tau^{\frac{\alpha}{k}}}{\left((1-\tau) b_{1}+\tau b_{2}\right)^{2}} \Upsilon^{\prime}\left(\frac{b_{1} b_{2}}{(1-\tau) b_{1}+\tau b_{2}}\right) \mathrm{d} \tau .
\end{aligned}
$$

Corollary 2.12 Choosing $\Phi(\tau)=\frac{\tau}{\alpha} \exp (-A \tau)$ in Lemma 2.4 with $A=\frac{1-\alpha}{\alpha}$ and $\alpha \in(0,1]$, then

$$
\begin{aligned}
& \Upsilon\left(\frac{2 b_{1} b_{2}}{b_{1}+b_{2}}\right)-\frac{1-\alpha}{2(1-\exp (-A))}\left(\frac{b_{1} b_{2}}{b_{2}-b_{1}}\right)^{\alpha}\left[I_{\frac{1}{b_{2}}}^{\alpha}+\Upsilon \circ \Psi\left(\frac{1}{b_{1}}\right)+I_{\frac{1}{b_{1}}}^{\alpha}-\Upsilon \circ \Psi\left(\frac{1}{b_{2}}\right)\right] \\
& \quad=\frac{b_{1} b_{2}\left(b_{2}-b_{1}\right)}{2(1-\exp (-A))} \sum_{j=13}^{16} L_{j},
\end{aligned}
$$

where

$$
\begin{aligned}
& L_{13}:=\int_{0}^{\frac{1}{2}} \frac{[\exp (-A \tau)-1]}{\left(\tau b_{1}+(1-\tau) b_{2}\right)^{2}} \Upsilon^{\prime}\left(\frac{b_{1} b_{2}}{\tau b_{1}+(1-\tau) b_{2}}\right) \mathrm{d} \tau, \\
& L_{14}:=\int_{0}^{\frac{1}{2}} \frac{[1-\exp (-A \tau)]}{\left((1-\tau) b_{1}+\tau b_{2}\right)^{2}} \Upsilon^{\prime}\left(\frac{b_{1} b_{2}}{(1-\tau) b_{1}+\tau b_{2}}\right) \mathrm{d} \tau \\
& L_{15}:=\int_{\frac{1}{2}}^{1} \frac{[\exp (-A(1-\tau))-\exp (-A \tau)]}{\left(\tau b_{1}+(1-\tau) b_{2}\right)^{2}} \Upsilon^{\prime}\left(\frac{b_{1} b_{2}}{\tau b_{1}+(1-\tau) b_{2}}\right) \mathrm{d} \tau, \\
& L_{16}:=\int_{\frac{1}{2}}^{1} \frac{[\exp (-A \tau)-\exp (-A(1-\tau))]}{\left((1-\tau) b_{1}+\tau b_{2}\right)^{2}} \Upsilon^{\prime}\left(\frac{b_{1} b_{2}}{(1-\tau) b_{1}+\tau b_{2}}\right) \mathrm{d} \tau .
\end{aligned}
$$

\subsection{Further results}

Now, utilizing auxiliary results obtained in the previous subsection, we derive some further generalized fractional trapezium-like inequalities using the class of harmonic convex functions. 
Theorem 2.5 Let $\Upsilon:\left[b_{1}, b_{2}\right] \rightarrow \mathbb{R}$ be a continuous function on $\left(b_{1}, b_{2}\right)$ with $b_{1}<b_{2}$ and $\left|\Upsilon^{\prime}\right|^{q}$ be an harmonic convex function with $\frac{1}{p}+\frac{1}{q}=1$, then

$$
\begin{aligned}
& \mid \frac{\Upsilon\left(b_{1}\right)+\Upsilon\left(b_{2}\right)}{m+1} \\
& \quad-\frac{1}{(m+1) \eta(1)}\left[\left(\frac{1}{b_{2}}\right)+I_{\Phi} \Upsilon \circ \Psi\left(\frac{m b_{1}+b_{2}}{(m+1) b_{1} b_{2}}\right)+{ }_{\left(\frac{1}{b_{1}}\right)-} I_{\Phi} \Upsilon \circ \Psi\left(\frac{b_{1}+m b_{2}}{(m+1) b_{1} b_{2}}\right)\right] \mid \\
& \quad \leq \frac{b_{1} b_{2}\left(b_{2}-b_{1}\right)}{\eta(1)}\left[\pi_{1}^{\frac{1}{p}}\left(\int_{0}^{1} \eta^{q}(\tau)\left(\frac{1-\tau}{m+1}\left|\Upsilon^{\prime}\left(b_{1}\right)\right|^{q}+\frac{m+\tau}{m+1}\left|\Upsilon^{\prime}\left(b_{2}\right)\right|^{q}\right) \mathrm{d} \tau\right)^{\frac{1}{q}}\right. \\
& \left.\quad+\pi_{2}^{\frac{1}{p}}\left(\int_{0}^{1} \eta^{q}(\tau)\left(\frac{m+\tau}{m+1}\left|\Upsilon^{\prime}\left(b_{1}\right)\right|^{q}+\frac{1-\tau}{m+1}\left|\Upsilon^{\prime}\left(b_{2}\right)\right|^{q}\right) \mathrm{d} \tau\right)^{\frac{1}{q}}\right],
\end{aligned}
$$

where

$$
\begin{aligned}
& \pi_{1}:=\frac{\left(m b_{1}+b_{2}\right)^{1-2 p}}{\left(b_{2}-b_{1}\right)(1-2 p)}\left[1-\left(\frac{(m+1) b_{1}}{m b_{1}+b_{2}}\right)^{1-2 p}\right], \\
& \pi_{2}:=\frac{\left(b_{1}+m b_{2}\right)^{1-2 p}}{\left(b_{2}-b_{1}\right)(1-2 p)}\left[\left(\frac{(m+1) b_{2}}{b_{1}+m b_{2}}\right)^{1-2 p}-1\right] .
\end{aligned}
$$

Proof Using Lemma 2.2, the modulus property, Hölder's inequality and the harmonic convexity of $\left|\Upsilon^{\prime}\right|^{q}$, we have

$$
\begin{aligned}
& \mid \frac{\Upsilon\left(b_{1}\right)+\Upsilon\left(b_{2}\right)}{m+1} \\
& \left.-\frac{1}{(m+1) \eta(1)}\left[\left(\frac{1}{b_{2}}\right)^{+} I_{\Phi} \Upsilon \circ \Psi\left(\frac{m b_{1}+b_{2}}{(m+1) b_{1} b_{2}}\right)+{ }_{\left(\frac{1}{b_{1}}\right)}\right) I_{\Phi} \Upsilon \circ \Psi\left(\frac{b_{1}+m b_{2}}{(m+1) b_{1} b_{2}}\right)\right] \mid \\
& \leq \frac{b_{1} b_{2}\left(b_{2}-b_{1}\right)}{\eta(1)}\left[\int_{0}^{1} \frac{\eta(\tau)}{\left((m+\tau) b_{1}+(1-\tau) b_{2}\right)^{2}}\left|\Upsilon^{\prime}\left(\frac{(m+1) b_{1} b_{2}}{(m+\tau) b_{1}+(1-\tau) b_{2}}\right)\right| \mathrm{d} \tau\right. \\
& \left.+\int_{0}^{1} \frac{\eta(\tau)}{\left((1-\tau) b_{1}+(m+\tau) b_{2}\right)^{2}}\left|\Upsilon^{\prime}\left(\frac{(m+1) b_{1} b_{2}}{(1-\tau) b_{1}+(m+\tau) b_{2}}\right)\right| \mathrm{d} \tau\right] \\
& \leq \frac{b_{1} b_{2}\left(b_{2}-b_{1}\right)}{\eta(1)}\left[\left(\int_{0}^{1}\left((m+\tau) b_{1}+(1-\tau) b_{2}\right)^{-2 p} \mathrm{~d} \tau\right)^{\frac{1}{p}}\right. \\
& \times\left(\int_{0}^{1} \eta^{p}(\tau)\left|\Upsilon^{\prime}\left(\frac{(m+1) b_{1} b_{2}}{(m+\tau) b_{1}+(1-\tau) b_{2}}\right)\right|^{q} \mathrm{~d} \tau\right)^{\frac{1}{q}} \\
& +\left(\int_{0}^{1}\left((1-\tau) b_{1}+(m+\tau) b_{2}\right)^{-2 p} \mathrm{~d} \tau\right)^{\frac{1}{p}} \\
& \left.\times\left(\int_{0}^{1} \eta^{q}(\tau)\left|\Upsilon^{\prime}\left(\frac{(m+1) b_{1} b_{2}}{(1-\tau) b_{1}+(m+\tau) b_{2}}\right)\right|^{q} \mathrm{~d} \tau\right)^{\frac{1}{q}}\right] \\
& \leq \frac{b_{1} b_{2}\left(b_{2}-b_{1}\right)}{\eta(1)}\left[\left(\int_{0}^{1}\left((m+\tau) b_{1}+(1-\tau) b_{2}\right)^{-2 p} \mathrm{~d} \tau\right)^{\frac{1}{p}}\right. \\
& \times\left(\int_{0}^{1} \eta^{q}(\tau)\left(\frac{1-\tau}{m+1}\left|\Upsilon^{\prime}\left(b_{1}\right)\right|^{q}+\frac{m+\tau}{m+1}\left|\Upsilon^{\prime}\left(b_{2}\right)\right|^{q}\right) \mathrm{d} \tau\right)^{\frac{1}{q}}
\end{aligned}
$$




$$
\begin{aligned}
& +\left(\int_{0}^{1}\left((1-\tau) b_{1}+(m+\tau) b_{2}\right)^{-2 p} \mathrm{~d} \tau\right)^{\frac{1}{p}} \\
& \left.\times\left(\int_{0}^{1} \eta^{q}(\tau)\left(\frac{m+\tau}{m+1}\left|\Upsilon^{\prime}\left(b_{1}\right)\right|^{q}+\frac{1-\tau}{m+1}\left|\Upsilon^{\prime}\left(b_{2}\right)\right|^{q}\right) \mathrm{d} \tau\right)^{\frac{1}{q}}\right] .
\end{aligned}
$$

After simple calculations, we obtain our required result.

Corollary 2.13 Choosing $\Phi(\tau)=\tau$ in Theorem 2.5, we have

$$
\begin{aligned}
& \left|\frac{\Upsilon\left(b_{1}\right)+\Upsilon\left(b_{2}\right)}{m+1}-\frac{b_{1} b_{2}}{b_{2}-b_{1}} \int_{\frac{1}{b_{2}}}^{\frac{1}{b_{1}}} \Upsilon \circ \Psi(x) \mathrm{d} x\right| \\
& \leq b_{1} b_{2}\left(b_{2}-b_{1}\right) \\
& \quad \times\left[\pi_{1}^{\frac{1}{p}}\left(\frac{1}{(m+1)(q+1)(q+2)}\left|\Upsilon^{\prime}\left(b_{1}\right)\right|^{q}+\frac{m(q+2)+(q+1)}{(m+1)(q+1)(q+2)}\left|\Upsilon^{\prime}\left(b_{2}\right)\right|^{q}\right)^{\frac{1}{q}}\right. \\
& \left.\quad+\pi_{2}^{\frac{1}{p}}\left(\frac{1}{(m+1)(q+1)(q+2)}\left|\Upsilon^{\prime}\left(b_{2}\right)\right|^{q}+\frac{m(q+2)+(q+1)}{(m+1)(q+1)(q+2)}\left|\Upsilon^{\prime}\left(b_{1}\right)\right|^{q}\right)^{\frac{1}{q}}\right],
\end{aligned}
$$

Corollary 2.14 Taking $\Phi(\tau)=\frac{\tau^{\alpha}}{\Gamma(\alpha)}$ in Theorem 2.5, we obtain

$$
\begin{aligned}
& \mid \frac{\Upsilon\left(b_{1}\right)+\Upsilon\left(b_{2}\right)}{m+1}-\frac{(m+1)^{\alpha-1}\left(b_{1} b_{2}\right)^{\alpha} \Gamma(\alpha+1)}{\left(b_{2}-b_{1}\right)^{\alpha}}\left[J_{\left(\frac{1}{b_{2}}\right)^{+}}^{\alpha} \Upsilon \circ \Psi\left(\frac{m b_{1}+b_{2}}{b_{1} b_{2}(m+1)}\right)\right. \\
& \left.\quad+J_{\left(\frac{1}{b_{1}}\right)^{-}}^{\alpha} \Upsilon \Psi\left(\frac{b_{1}+m b_{2}}{(m+1) b_{1} b_{2}}\right)\right] \mid \\
& \leq b_{1} b_{2}\left(b_{2}-b_{1}\right)\left[\pi _ { 1 } ^ { \frac { 1 } { p } } \left(\frac{1}{(m+1)(\alpha q+1)(\alpha q+2)}\left|\Upsilon^{\prime}\left(b_{1}\right)\right|^{q}\right.\right. \\
& \left.\quad+\frac{m(\alpha q+2)+(\alpha q+1)}{(m+1)(\alpha q+1)(\alpha q+2)}\left|\Upsilon^{\prime}\left(b_{2}\right)\right|^{q}\right)^{\frac{1}{q}} \\
& \quad+\pi_{2}^{\frac{1}{p}}\left(\frac{1}{(m+1)(\alpha q+1)(\alpha q+2)}\left|\Upsilon^{\prime}\left(b_{2}\right)\right|^{q}\right. \\
& \left.\left.\quad+\frac{m(\alpha q+2)+(\alpha q+1)}{(m+1)(\alpha q+1)(\alpha q+2)}\left|\Upsilon^{\prime}\left(b_{1}\right)\right|^{q}\right)^{\frac{1}{q}}\right],
\end{aligned}
$$

where $\pi_{1}$ and $\pi_{2}$ are already defined.

Corollary 2.15 Choosing $\Phi(\tau)=\frac{\tau^{\frac{\alpha}{k}}}{k \Gamma_{k}(\alpha)}$ in Theorem 2.5, we obtain

$$
\begin{aligned}
& \mid \frac{\Upsilon\left(b_{1}\right)+\Upsilon\left(b_{2}\right)}{m+1}-\frac{(m+1)^{\frac{\alpha}{k}-1}\left(b_{1} b_{2}\right)^{\frac{\alpha}{k}} \Gamma_{k}(\alpha+k)}{\left(b_{2}-b_{1}\right)^{\frac{\alpha}{k}}}\left[k_{\left(\frac{1}{b_{2}}\right)^{+}}^{\alpha} \Upsilon \circ \Psi\left(\frac{m b_{1}+b_{2}}{b_{1} b_{2}(m+1)}\right)\right. \\
& \left.\quad+{ }_{k} J_{\left(\frac{1}{b_{1}}\right)^{-}}^{\alpha} \Upsilon \circ \Psi\left(\frac{b_{1}+m b_{2}}{(m+1) b_{1} b_{2}}\right)\right] \mid \\
& \leq b_{1} b_{2}\left(b_{2}-b_{1}\right)\left[\pi _ { 1 } ^ { \frac { 1 } { p } } \left(\frac{k}{(m+1)(\alpha q+k)(\alpha q+2 k)}\left|\Upsilon^{\prime}\left(b_{1}\right)\right|^{q}\right.\right. \\
& \left.\quad+\frac{k m(\alpha q+2 k)+k(\alpha q+k)}{(m+1)(\alpha q+k)(\alpha q+2 k)}\left|\Upsilon^{\prime}\left(b_{2}\right)\right|^{q}\right)^{\frac{1}{q}}
\end{aligned}
$$




$$
\begin{aligned}
& +\pi_{2}^{\frac{1}{p}}\left(\frac{k}{(m+1)(\alpha q+k)(\alpha q+2 k)}\left|\Upsilon^{\prime}\left(b_{2}\right)\right|^{q}\right. \\
& \left.\left.+\frac{m k(\alpha q+2 k)+(k \alpha q+k)}{(m+1)(\alpha q+k)(\alpha q+2 k)}\left|\Upsilon^{\prime}\left(b_{1}\right)\right|^{q}\right)^{\frac{1}{q}}\right] .
\end{aligned}
$$

Theorem 2.6 Let $\Upsilon:\left[b_{1}, b_{2}\right] \rightarrow \mathbb{R}$ be a continuous function on $\left(b_{1}, b_{2}\right)$ with $b_{1}<b_{2}$ and $\left|\Upsilon^{\prime}\right|^{q}$ be an harmonic convex function with $q \geq 1$, then

$$
\begin{aligned}
& \mid \frac{\Upsilon\left(b_{1}\right)+\Upsilon\left(b_{2}\right)}{m+1} \\
& \quad-\frac{1}{(m+1) \eta(1)}\left[\left(\frac{1}{b_{2}}\right)+I_{\Phi} \Upsilon \circ \Psi\left(\frac{m b_{1}+b_{2}}{(m+1) b_{1} b_{2}}\right)+{ }_{\left(\frac{1}{b_{1}}\right)-} I_{\Phi} \Upsilon \circ \Psi\left(\frac{b_{1}+m b_{2}}{(m+1) b_{1} b_{2}}\right)\right] \mid \\
& \quad \leq \frac{b_{1} b_{2}\left(b_{2}-b_{1}\right)}{\eta(1)}\left[\left(\int_{0}^{1} \eta(\tau)\left((m+\tau) b_{1}+(1-\tau) b_{2}\right)^{-2} \mathrm{~d} \tau\right)^{1-\frac{1}{q}}\right. \\
& \quad \times\left(\int_{0}^{1} \eta(\tau)\left((m+\tau) b_{1}+(1-\tau) b_{2}\right)^{-2}\left(\frac{1-\tau}{m+1}\left|\Upsilon^{\prime}\left(b_{1}\right)\right|^{q}+\frac{m+\tau}{m+1}\left|\Upsilon^{\prime}\left(b_{2}\right)\right|^{q}\right) \mathrm{d} \tau\right)^{\frac{1}{q}} \\
& \quad+\left(\int_{0}^{1} \eta(\tau)\left((1-\tau) b_{1}+(m+\tau) b_{2}\right)^{-2} \mathrm{~d} \tau\right)^{1-\frac{1}{q}} \\
& \quad \times\left(\int_{0}^{1} \eta(\tau)\left((1-\tau) b_{1}+(m+\tau) b_{2}\right)^{-2}\right. \\
& \left.\left.\quad \times\left(\frac{m+\tau}{m+1}\left|\Upsilon^{\prime}\left(b_{1}\right)\right|^{q}+\frac{1-\tau}{m+1}\left|\Upsilon^{\prime}\left(b_{2}\right)\right|^{q}\right) \mathrm{d} \tau\right)^{\frac{1}{q}}\right] .
\end{aligned}
$$

Proof Using Lemma 2.2, the modulus property, the power mean inequality and the convexity of $\left|\Upsilon^{\prime}\right|^{q}$, we have

$$
\begin{aligned}
& \mid \frac{\Upsilon\left(b_{1}\right)+\Upsilon\left(b_{2}\right)}{m+1} \\
& \quad-\frac{1}{(m+1) \eta(1)}\left[\left(\frac{1}{b_{2}}\right)^{+} I_{\Phi} \Upsilon \circ \Psi\left(\frac{m b_{1}+b_{2}}{(m+1) b_{1} b_{2}}\right)+{ }_{\left(\frac{1}{b_{1}}\right)-} I_{\Phi} \Upsilon \circ \Psi\left(\frac{b_{1}+m b_{2}}{(m+1) b_{1} b_{2}}\right)\right] \mid \\
& \leq \leq \frac{b_{1} b_{2}\left(b_{2}-b_{1}\right)}{\eta(1)}\left[\int_{0}^{1} \frac{\eta(\tau)}{\left((m+\tau) b_{1}+(1-\tau) b_{2}\right)^{2}}\left|\Upsilon^{\prime}\left(\frac{(m+1) b_{1} b_{2}}{(m+\tau) b_{1}+(1-\tau) b_{2}}\right)\right| \mathrm{d} \tau\right. \\
& \left.\quad+\int_{0}^{1} \frac{\eta(\tau)}{\left((1-\tau) b_{1}+(m+\tau) b_{2}\right)^{2}}\left|\Upsilon^{\prime}\left(\frac{(m+1) b_{1} b_{2}}{(1-\tau) b_{1}+(m+\tau) b_{2}}\right)\right| \mathrm{d} \tau\right] \\
& \leq \\
& \quad \times\left(\int_{0}^{1} \eta(\tau)\left((m+\tau) b_{1}+(1-\tau) b_{2}\right)^{-2}\left|\Upsilon^{\prime}\left(\frac{(m+1) b_{1} b_{2}}{(m+\tau) b_{1}+(1-\tau) b_{2}}\right)\right|^{q} \mathrm{~d} \tau\right)^{\frac{1}{q}} \\
& \quad+\left(\int_{0}^{1} \eta(\tau)\left((m+\tau) b_{1}+(1-\tau) b_{2}\right)^{-2} \mathrm{~d} \tau\right)^{1-\frac{1}{q}} \\
& \quad \\
& \quad \times\left(\int_{0}^{1} \eta(\tau)\left((1-\tau) b_{1}+(m+\tau) b_{2}\right)^{-2} \mathrm{~d} \tau\right)^{1-\frac{1}{q}}
\end{aligned}
$$




$$
\begin{aligned}
\leq & \frac{b_{1} b_{2}\left(b_{2}-b_{1}\right)}{\eta(1)}\left[\left(\int_{0}^{1} \eta(\tau)\left((m+\tau) b_{1}+(1-\tau) b_{2}\right)^{-2} \mathrm{~d} \tau\right)^{1-\frac{1}{q}}\right. \\
& \times\left(\int_{0}^{1} \eta(\tau)\left((m+\tau) b_{1}+(1-\tau) b_{2}\right)^{-2}\left(\frac{1-\tau}{m+1}\left|\Upsilon^{\prime}\left(b_{1}\right)\right|^{q}+\frac{m+\tau}{m+1}\left|\Upsilon^{\prime}\left(b_{2}\right)\right|^{q}\right) \mathrm{d} \tau\right)^{\frac{1}{q}} \\
& +\left(\int_{0}^{1} \eta(\tau)\left((1-\tau) b_{1}+(m+\tau) b_{2}\right)^{-2}\right)^{1-\frac{1}{q}} \\
& \times\left(\int_{0}^{1} \eta(\tau)\left((1-\tau) b_{1}+(m+\tau) b_{2}\right)^{-2}\right. \\
& \left.\left.\times\left(\frac{m+\tau}{m+1}\left|\Upsilon^{\prime}\left(b_{1}\right)\right|^{q}+\frac{1-\tau}{m+1}\left|\Upsilon^{\prime}\left(b_{2}\right)\right|^{q}\right) \mathrm{d} \tau\right)^{\frac{1}{q}}\right]
\end{aligned}
$$

After simple calculations, we obtain our required result.

Corollary 2.16 If we take $\Phi(\tau)=\tau$ in Theorem 2.6, we have

$$
\begin{aligned}
& \left|\frac{\Upsilon\left(b_{1}\right)+\Upsilon\left(b_{2}\right)}{m+1}-\frac{b_{1} b_{2}}{b_{2}-b_{1}} \int_{\frac{1}{b_{2}}}^{\frac{1}{b_{1}}} \Upsilon \circ \Psi(x) \mathrm{d} x\right| \\
& \leq b_{1} b_{2}\left(b_{2}-b_{1}\right)\left[\pi_{3}^{1-\frac{1}{q}}\left(\pi_{4}\left|\Upsilon^{\prime}\left(b_{1}\right)\right|^{q}+\pi_{5}\left|\Upsilon^{\prime}\left(b_{2}\right)\right|^{q}\right)^{\frac{1}{q}}\right. \\
& \left.\quad+\pi_{6}^{1-\frac{1}{q}}\left(\pi_{7}\left|\Upsilon^{\prime}\left(b_{1}\right)\right|^{q}+\pi_{8}\left|\Upsilon^{\prime}\left(b_{2}\right)\right|^{q}\right)^{\frac{1}{q}}\right],
\end{aligned}
$$

where

$$
\begin{aligned}
& \pi_{3}:=\frac{\left(m b_{1}+b_{2}\right)^{-2}}{2}{ }_{2} F_{1}\left(2,2,3, \frac{b_{2}-b_{1}}{m b_{1}+b_{2}}\right), \\
& \pi_{4}:=\frac{\left(m b_{1}+b_{2}\right)^{-2}}{6(m+1)}{ }_{2} F_{1}\left(2,2,4, \frac{b_{2}-b_{1}}{m b_{1}+b_{2}}\right), \\
& \pi_{5}:=\frac{m\left(m b_{1}+b_{2}\right)^{-2}}{2(m+1)}{ }_{2} F_{1}\left(2,2,3, \frac{b_{2}-b_{1}}{m b_{1}+b_{2}}\right)+\frac{\left(m b_{1}+b_{2}\right)^{-2}}{6(m+1)}{ }_{2} F_{1}\left(2,3,4, \frac{b_{2}-b_{1}}{m b_{1}+b_{2}}\right), \\
& \pi_{6}:=\frac{\left(b_{1}+m b_{2}\right)^{-2}}{2}{ }_{2} F_{1}\left(2,2,3, \frac{b_{1}-b_{2}}{b_{1}+m b_{2}}\right), \\
& \pi_{7}:=\frac{m\left(b_{1}+m b_{2}\right)^{-2}}{2(m+1)}{ }_{2} F_{1}\left(2,2,3, \frac{b_{1}-b_{2}}{b_{1}+m b_{2}}\right)+\frac{\left(b_{1}+m b_{2}\right)^{-2}}{6(m+1)}{ }_{2} F_{1}\left(2,3,4, \frac{b_{1}-b_{2}}{b_{1}+m b_{2}}\right), \\
& \pi_{8}:=\frac{\left(b_{1}+m b_{2}\right)^{-2}}{6(m+1)}{ }_{2} F_{1}\left(2,2,4, \frac{b_{1}-b_{2}}{b_{1}+m b_{2}}\right) .
\end{aligned}
$$

Corollary 2.17 If we choose $\Phi(\tau)=\frac{\tau^{\alpha}}{\Gamma(\alpha)}$ in Theorem 2.6, we obtain

$$
\begin{aligned}
& \mid \frac{\Upsilon\left(b_{1}\right)+\Upsilon\left(b_{2}\right)}{m+1}-\frac{(m+1)^{\alpha-1}\left(b_{1} b_{2}\right)^{\alpha} \Gamma(\alpha+1)}{\left(b_{2}-b_{1}\right)^{\alpha}}\left[J_{\left(\frac{1}{b_{2}}\right)^{+}}^{\alpha} \Upsilon \circ \Psi\left(\frac{m b_{1}+b_{2}}{b_{1} b_{2}(m+1)}\right)\right. \\
& \left.\quad+J_{\left(\frac{1}{b_{1}}\right)^{-}}^{\alpha} \Upsilon \circ \Psi\left(\frac{b_{1}+m b_{2}}{(m+1) b_{1} b_{2}}\right)\right] \mid
\end{aligned}
$$




$$
\begin{aligned}
\leq & b_{1} b_{2}\left(b_{2}-b_{1}\right)\left[\pi_{9}^{1-\frac{1}{q}}\left(\pi_{10}\left|\Upsilon^{\prime}\left(b_{1}\right)\right|^{q}+\pi_{11}\left|\Upsilon^{\prime}\left(b_{2}\right)\right|^{q}\right)^{\frac{1}{q}}\right. \\
& \left.+\pi_{12}^{1-\frac{1}{q}}\left(\pi_{13}\left|\Upsilon^{\prime}\left(b_{1}\right)\right|^{q}+\pi_{14}\left|\Upsilon^{\prime}\left(b_{2}\right)\right|^{q}\right)^{\frac{1}{q}}\right]
\end{aligned}
$$

where

$$
\begin{aligned}
\pi_{9}:= & \frac{\left(m b_{1}+b_{2}\right)^{-2}}{\alpha+1}{ }_{2} F_{1}\left(2, \alpha+1, \alpha+2, \frac{b_{2}-b_{1}}{m b_{1}+b_{2}}\right), \\
\pi_{10}:= & \frac{\left(m b_{1}+b_{2}\right)^{-2}}{(\alpha+2)(\alpha+1)(m+1)}{ }_{2} F_{1}\left(2, \alpha+1, \alpha+3, \frac{b_{2}-b_{1}}{m b_{1}+b_{2}}\right), \\
\pi_{11}:= & \frac{m\left(m b_{1}+b_{2}\right)^{-2}}{(\alpha+1)(m+1)}{ }_{2} F_{1}\left(2, \alpha+1, \alpha+2, \frac{b_{2}-b_{1}}{m b_{1}+b_{2}}\right) \\
& +\frac{\left(m b_{1}+b_{2}\right)^{-2}}{(\alpha+2)(\alpha+1)(m+1)}{ }_{2} F_{1}\left(2, \alpha+2, \alpha+3, \frac{b_{2}-b_{1}}{m b_{1}+b_{2}}\right), \\
\pi_{12}:= & \frac{\left(b_{1}+m b_{2}\right)^{-2}}{\alpha+1}{ }_{2} F_{1}\left(2, \alpha+1, \alpha+2, \frac{b_{1}-b_{2}}{b_{1}+m b_{2}}\right), \\
\pi_{13}:= & \frac{m\left(b_{1}+m b_{2}\right)^{-2}}{(\alpha+1)(m+1)}{ }_{2} F_{1}\left(2, \alpha+1, \alpha+2, \frac{b_{1}-b_{2}}{b_{1}+m b_{2}}\right) \\
& +\frac{\left(b_{1}+m b_{2}\right)^{-2}}{(\alpha+2)(\alpha+1)(m+1)}{ }_{2} F_{1}\left(2, \alpha+2, \alpha+3, \frac{b_{1}-b_{2}}{b_{1}+m b_{2}}\right), \\
\pi_{14}:= & \frac{\left(b_{1}+m b_{2}\right)^{-2}}{(\alpha+2)(\alpha+1)(m+1)}{ }_{2} F_{1}\left(2, \alpha+1, \alpha+3, \frac{b_{1}-b_{2}}{b_{1}+m b_{2}}\right) .
\end{aligned}
$$

Corollary 2.18 If we take $\Phi(\tau)=\frac{\tau^{\frac{\alpha}{k}}}{k \Gamma_{k}(\alpha)}$ in Theorem 2.6, we obtain

$$
\begin{aligned}
& \mid \frac{\Upsilon\left(b_{1}\right)+\Upsilon\left(b_{2}\right)}{m+1}-\frac{(m+1)^{\frac{\alpha}{k}-1}\left(b_{1} b_{2}\right)^{\frac{\alpha}{k}} \Gamma_{k}(\alpha+k)}{\left(b_{2}-b_{1}\right)^{\frac{\alpha}{k}}}\left[{ }_{k} J_{\left(\frac{1}{b_{2}}\right)^{+}}^{\alpha} \Upsilon \circ \Psi\left(\frac{m b_{1}+b_{2}}{b_{1} b_{2}(m+1)}\right)\right. \\
& \left.\quad+{ }_{k} J_{\left(\frac{1}{b_{1}}\right)^{-}}^{\alpha} \Upsilon \circ \Psi\left(\frac{b_{1}+m b_{2}}{(m+1) b_{1} b_{2}}\right)\right] \mid \\
& \quad \leq b_{1} b_{2}\left(b_{2}-b_{1}\right)\left[\pi_{15}^{1-\frac{1}{q}}\left(\pi_{16}\left|\Upsilon^{\prime}\left(b_{1}\right)\right|^{q}+\pi_{17}\left|\Upsilon^{\prime}\left(b_{2}\right)\right|^{q}\right)^{\frac{1}{q}}\right. \\
& \left.\quad+\pi_{18}^{1-\frac{1}{q}}\left(\pi_{19}\left|\Upsilon^{\prime}\left(b_{1}\right)\right|^{q}+\pi_{20}\left|\Upsilon^{\prime}\left(b_{2}\right)\right|^{q}\right)^{\frac{1}{q}}\right],
\end{aligned}
$$

where

$$
\begin{aligned}
\pi_{15}:= & \frac{k\left(m b_{1}+b_{2}\right)^{-2}}{\alpha+k}{ }_{2} F_{1, k}\left(2 k, \alpha+k, \alpha+2 k, \frac{b_{2}-b_{1}}{m b_{1}+b_{2}}\right), \\
\pi_{16}:= & \frac{k\left(m b_{1}+b_{2}\right)^{-2}}{(\alpha+2 k)(\alpha+k)(m+1)}{ }_{2} F_{1, k}\left(2 k, \alpha+k, \alpha+3 k, \frac{b_{2}-b_{1}}{m b_{1}+b_{2}}\right), \\
\pi_{17}:= & \frac{k m\left(m b_{1}+b_{2}\right)^{-2}}{(\alpha+k)(m+1)}{ }_{2} F_{1, k}\left(2 k, \alpha+k, \alpha+2 k, \frac{b_{2}-b_{1}}{m b_{1}+b_{2}}\right) \\
& +\frac{k\left(m b_{1}+b_{2}\right)^{-2}}{(\alpha+2 k)(\alpha+k)(m+1)}{ }_{2} F_{1, k}\left(2 k, \alpha+2 k, \alpha+3 k, \frac{b_{2}-b_{1}}{m b_{1}+b_{2}}\right), \\
\pi_{18}:= & \frac{k\left(b_{1}+m b_{2}\right)^{-2}}{\alpha+k}{ }_{2} F_{1, k}\left(2 k, \alpha+k, \alpha+2 k, \frac{b_{1}-b_{2}}{b_{1}+m b_{2}}\right)
\end{aligned}
$$




$$
\begin{aligned}
\pi_{19}:= & \frac{k m\left(b_{1}+m b_{2}\right)^{-2}}{(\alpha+k)(m+1)}{ }_{2} F_{1, k}\left(2 k, \alpha+k, \alpha+2 k, \frac{b_{1}-b_{2}}{b_{1}+m b_{2}}\right) \\
& +\frac{k\left(b_{1}+m b_{2}\right)^{-2}}{(\alpha+2 k)(\alpha+k)(m+1)}{ }_{2} F_{1, k}\left(2 k, \alpha+2 k, \alpha+3 k, \frac{b_{1}-b_{2}}{b_{1}+m b_{2}}\right), \\
\pi_{20}:= & \frac{k\left(b_{1}+m b_{2}\right)^{-2}}{(\alpha+2 k)(\alpha+k)(m+1)}{ }_{2} F_{1, k}\left(2 k, \alpha+k, \alpha+3 k, \frac{b_{1}-b_{2}}{b_{1}+m b_{2}}\right) .
\end{aligned}
$$

Theorem 2.7 Let $\Upsilon:\left[b_{1}, b_{2}\right] \rightarrow \mathbb{R}$ be a continuous function on $\left(b_{1}, b_{2}\right)$ with $b_{1}<b_{2}$ and $\left|\Upsilon^{\prime}\right|^{q}$ be an harmonic convex function with $\frac{1}{p}+\frac{1}{q}=1$ and $\lambda, \mu \in[0, \infty)$ with $\lambda+\mu \neq 0$, then

$$
\begin{aligned}
& \mid \frac{\Omega(\lambda) \Upsilon\left(b_{2}\right)+\Omega(\mu) \Upsilon\left(b_{1}\right)}{\lambda+\mu} \\
& \quad-\frac{1}{\lambda+\mu}\left[\left(\frac{1}{\left.b_{2}\right)^{+}} I_{\Phi} \Upsilon \circ \Psi\left(\frac{\lambda b_{2}+\mu b_{1}}{b_{1} b_{2}(\lambda+\mu)}\right)+{ }_{\left(\frac{1}{b_{1}}\right)^{-}} I_{\Phi} \Upsilon \circ \Psi\left(\frac{\lambda b_{2}+\mu b_{1}}{b_{1} b_{2}(\lambda+\mu)}\right)\right] \mid\right. \\
& \quad \leq b_{1} b_{2}\left(b_{2}-b_{1}\right)\left[\left(\int_{0}^{\lambda} \Omega^{p}(\tau) \mathrm{d} \tau\right)^{\frac{1}{p}}\left(\sigma_{1}\left|\Upsilon^{\prime}\left(b_{2}\right)\right|^{q}+\sigma_{2}\left|\Upsilon^{\prime}\left(b_{1}\right)\right|^{q}\right)^{\frac{1}{q}}\right. \\
& \left.\quad+\left(\int_{0}^{\mu} \Omega^{p}(\tau) \mathrm{d} \tau\right)^{\frac{1}{p}}\left(\sigma_{3}\left|\Upsilon^{\prime}\left(b_{2}\right)\right|^{q}+\sigma_{4}\left|\Upsilon^{\prime}\left(b_{1}\right)\right|^{q}\right)^{\frac{1}{q}}\right]
\end{aligned}
$$

where

$$
\begin{aligned}
\sigma_{1} & :=\int_{0}^{\lambda} \frac{\left((\lambda-\tau) b_{2}+(\mu+\tau) b_{1}\right)^{-2 q}(\mu+\tau)}{\lambda+\mu} \mathrm{d} \tau \\
& =\frac{\mu\left(\lambda b_{2}+\mu b_{1}\right)^{1-2 q}-(\lambda+\mu)\left((\lambda+\mu) b_{1}\right)^{1-2 q}}{(\lambda+\mu)\left(b_{2}-b_{1}\right)(1-2 q)}-\frac{\left((\lambda+\mu) b_{1}\right)^{2-2 q}-\left(\lambda b_{2}+\mu b_{1}\right)^{2-2 q}}{(\lambda+\mu)\left(b_{2}-b_{1}\right)^{2}(1-2 q)(2-2 q)}, \\
\sigma_{2} & :=\int_{0}^{\lambda} \frac{\left((\lambda-\tau) b_{2}+(\mu+\tau) b_{1}\right)^{-2 q}(\lambda-\tau)}{\lambda+\mu} \mathrm{d} \tau \\
& =\frac{\lambda\left(\lambda b_{2}+\mu b_{1}\right)^{1-2 q}}{(\lambda+\mu)\left(b_{2}-b_{1}\right)(1-2 q)}+\frac{\left((\lambda+\mu) b_{1}\right)^{2-2 q}-\left(\lambda b_{2}+\mu b_{1}\right)^{2-2 q}}{(\lambda+\mu)\left(b_{2}-b_{1}\right)^{2}(1-2 q)(2-2 q)}, \\
\sigma_{3} & :=\int_{0}^{\mu} \frac{\left((\lambda+\tau) b_{2}+(\mu-\tau) b_{1}\right)^{-2 q}(\mu-\tau)}{\lambda+\mu} \mathrm{d} \tau \\
& =\frac{\left((\lambda+\mu) b_{2}\right)^{2-2 q}-\left(\lambda b_{2}+\mu b_{1}\right)^{2-2 q}}{(\lambda+\mu)\left(b_{2}-b_{1}\right)^{2}(1-2 q)(2-2 q)} \frac{\mu\left(\lambda b_{2}+\mu b_{1}\right)^{1-2 q}}{(\lambda+\mu)\left(b_{2}-b_{1}\right)(1-2 q)}, \\
\sigma_{4} & :=\int_{0}^{\mu} \frac{\left((\lambda+\tau) b_{2}+(\mu-\tau) b_{1}\right)^{-2 q}(\lambda+\tau)}{\lambda+\mu} \mathrm{d} \tau \\
& =\frac{(\lambda+\mu)\left((\lambda+\mu) b_{2}\right)^{1-2 q}-\lambda\left(\lambda b_{2}+\mu b_{1}\right)^{1-2 q}}{(\lambda+\mu)\left(b_{2}-b_{1}\right)(1-2 q)}-\frac{\left((\lambda+\mu) b_{2}\right)^{2-2 q}-\left(\lambda b_{2}+\mu b_{1}\right)^{2-2 q}}{(\lambda+\mu)\left(b_{2}-b_{1}\right)^{2}(1-2 q)(2-2 q)} .
\end{aligned}
$$

Proof Using Lemma 2.3, the modulus property, Hölder's inequality and the harmonic convexity of $\left|\Upsilon^{\prime}\right|^{q}$, we have

$$
\begin{aligned}
& \mid \frac{\Omega(\lambda) \Upsilon\left(b_{2}\right)+\Omega(\mu) \Upsilon\left(b_{1}\right)}{\lambda+\mu} \\
& \quad-\frac{1}{\lambda+\mu}\left[\left(\frac{1}{b_{2}}\right)^{+} I_{\Phi} \Upsilon \circ \Psi\left(\frac{\lambda b_{2}+\mu b_{1}}{b_{1} b_{2}(\lambda+\mu)}\right)+{ }_{\left(\frac{1}{b_{1}}\right)^{-}} I_{\Phi} \Upsilon \circ \Psi\left(\frac{\lambda b_{2}+\mu b_{1}}{b_{1} b_{2}(\lambda+\mu)}\right)\right] \mid
\end{aligned}
$$




$$
\begin{aligned}
\leq & b_{1} b_{2}\left(b_{2}-b_{1}\right)\left[\left(\int_{0}^{\lambda} \Omega^{p}(\tau) \mathrm{d} \tau\right)^{\frac{1}{p}}\right. \\
& \times\left(\int_{0}^{\lambda} \frac{1}{\left((\lambda-\tau) b_{2}+(\mu+\tau) b_{1}\right)^{2 p}}\left|\Upsilon^{\prime}\left(\frac{b_{1} b_{2}(\lambda+\mu)}{(\lambda-\tau) b_{2}+(\mu+\tau) b_{1}}\right)\right|^{q} \mathrm{~d} \tau\right)^{\frac{1}{q}} \\
& +\left(\int_{0}^{\mu} \Omega^{p}(\tau) \mathrm{d} \tau\right)^{\frac{1}{p}} \\
& \left.\times\left(\int_{0}^{\mu} \frac{1}{\left((\lambda+\tau) b_{2}+(\mu-\tau) b_{1}\right)^{2 p}}\left|\Upsilon^{\prime}\left(\frac{b_{1} b_{2}(\lambda+\mu)}{(\lambda+\tau) b_{2}+(\mu-\tau) b_{1}}\right)\right|^{q} \mathrm{~d} \tau\right)^{\frac{1}{q}}\right] \\
\leq & b_{1} b_{2}\left(b_{2}-b_{1}\right)\left[( \int _ { 0 } ^ { \lambda } \Omega ^ { p } ( \tau ) \mathrm { d } \tau ) ^ { \frac { 1 } { p } } \left(\int_{0}^{\lambda}\left((\lambda-\tau) b_{2}+(\mu+\tau) b_{1}\right)^{-2 q}\right.\right. \\
& \left.\times\left(\frac{\mu+\tau}{\lambda+\mu}\left|\Upsilon^{\prime}\left(b_{2}\right)\right|^{q}+\frac{\lambda-\tau}{\lambda+\mu}\left|\Upsilon^{\prime}\left(b_{1}\right)\right|^{q}\right) \mathrm{d} \tau\right)^{\frac{1}{q}} \\
& +\left(\int_{0}^{\mu} \Omega^{p}(\tau) \mathrm{d} \tau\right)^{\frac{1}{p}}\left(\int_{0}^{\mu}\left((\lambda+\tau) b_{2}+(\mu-\tau) b_{1}\right)^{-2 q}\right. \\
& \left.\left.\times\left(\frac{\mu-\tau}{\lambda+\mu}\left|\Upsilon^{\prime}\left(b_{2}\right)\right|^{q}+\frac{\lambda+\tau}{\lambda+\mu}\left|\Upsilon^{\prime}\left(b_{1}\right)\right|^{q}\right) \mathrm{d} \tau\right)^{\frac{1}{q}}\right] .
\end{aligned}
$$

After simple calculations, we obtain our required result.

Corollary 2.19 Choosing $\Phi(\tau)=\tau$ in Theorem 2.7, we have

$$
\begin{aligned}
& \left|\frac{\lambda \Upsilon\left(b_{2}\right)+\mu \Upsilon\left(b_{1}\right)}{\lambda+\mu}-\frac{b_{1} b_{2}}{b_{2}-b_{1}} \int_{\frac{1}{b_{2}}}^{\frac{1}{b_{1}}} \Upsilon \circ \Psi(x) \mathrm{d} x\right| \\
& \leq b_{1} b_{2}\left(b_{2}-b_{1}\right)\left[\left(\frac{\lambda^{p+1}}{p+1}\right)^{\frac{1}{p}}\left(\sigma_{1}\left|\Upsilon^{\prime}\left(b_{2}\right)\right|^{q}+\sigma_{2}\left|\Upsilon^{\prime}\left(b_{1}\right)\right|^{q}\right)^{\frac{1}{q}}+\left(\frac{\mu^{p+1}}{p+1}\right)^{\frac{1}{p}}\right. \\
& \left.\quad \times\left(\sigma_{3}\left|\Upsilon^{\prime}\left(b_{2}\right)\right|^{q}+\sigma_{4}\left|\Upsilon^{\prime}\left(b_{1}\right)\right|^{q}\right)^{\frac{1}{q}}\right]
\end{aligned}
$$

where $\sigma_{1}, \sigma_{2}, \sigma_{3}$ and $\sigma_{4}$ are already defined in Theorem 2.7.

Corollary 2.20 Taking $\Phi(\tau)=\frac{\tau^{\alpha}}{\Gamma(\alpha)}$ in Theorem 2.7, we obtain

$$
\begin{aligned}
& \mid \frac{\lambda^{\alpha} \Upsilon\left(b_{2}\right)+\mu^{\alpha} \Upsilon\left(b_{1}\right)}{\lambda+\mu}-\frac{\left(b_{1} b_{2}\right)^{\alpha}(\lambda+\mu)^{\alpha-1} \Gamma(\alpha+1)}{\left(b_{2}-b_{1}\right)^{\alpha}}\left[J_{\left(\frac{1}{b_{2}}\right)^{+}}^{\alpha} \Upsilon \circ \Psi\left(\frac{\lambda b_{2}+\mu b_{1}}{b_{1} b_{2}(\lambda+\mu)}\right)\right. \\
& \left.\quad+J_{\left(\frac{1}{b_{1}}\right)^{-}}^{\alpha} \Upsilon \circ \Psi\left(\frac{\lambda b_{2}+\mu b_{1}}{b_{1} b_{2}(\lambda+\mu)}\right)\right] \mid \\
& \leq b_{1} b_{2}\left(b_{2}-b_{1}\right)\left[\left(\frac{\lambda^{\alpha p+1}}{\alpha p+1}\right)^{\frac{1}{p}}\left(\sigma_{1}\left|\Upsilon^{\prime}\left(b_{2}\right)\right|^{q}+\sigma_{2}\left|\Upsilon^{\prime}\left(b_{1}\right)\right|^{q}\right)^{\frac{1}{q}}+\left(\frac{\mu^{\alpha p+1}}{\alpha p+1}\right)^{\frac{1}{p}}\right. \\
& \left.\quad \times\left(\sigma_{3}\left|\Upsilon^{\prime}\left(b_{2}\right)\right|^{q}+\sigma_{4}\left|\Upsilon^{\prime}\left(b_{1}\right)\right|^{q}\right)^{\frac{1}{q}}\right],
\end{aligned}
$$

where $\sigma_{1}, \sigma_{2}, \sigma_{3}$ and $\sigma_{4}$ are already defined in Theorem 2.7. 
Corollary 2.21 Choosing $\Phi(\tau)=\frac{\tau^{\frac{\alpha}{k}}}{k \Gamma_{k}(\alpha)}$ in Theorem 2.7, we obtain

$$
\begin{aligned}
& \mid \frac{\lambda^{\frac{\alpha}{k}} \Upsilon\left(b_{2}\right)+\mu^{\frac{\alpha}{k}} \Upsilon\left(b_{1}\right)}{\lambda+\mu}-\frac{k\left(b_{1} b_{2}\right)^{\frac{\alpha}{k}}(\lambda+\mu)^{\frac{\alpha}{k}-1} \Gamma_{k}(\alpha+k)}{\left(b_{2}-b_{1}\right)^{\frac{\alpha}{k}}}\left[{ }_{k} J_{\left(\frac{1}{b_{2}}\right)^{+}}^{\alpha} \Upsilon \circ \Psi\left(\frac{\lambda b_{2}+\mu b_{1}}{b_{1} b_{2}(\lambda+\mu)}\right)\right. \\
& \left.\quad+{ }_{k} J_{\left(\frac{1}{b_{1}}\right)^{-}}^{\alpha} \Upsilon \circ \Psi\left(\frac{\lambda b_{2}+\mu b_{1}}{b_{1} b_{2}(\lambda+\mu)}\right)\right] \mid \\
& \quad \leq b_{1} b_{2}\left(b_{2}-b_{1}\right)\left[\left(\frac{k \lambda \frac{\alpha p+k}{k}}{\alpha p+k}\right)^{\frac{1}{p}}\left(\sigma_{1}\left|\Upsilon^{\prime}\left(b_{2}\right)\right|^{q}+\sigma_{2}\left|\Upsilon^{\prime}\left(b_{1}\right)\right|^{q}\right)^{\frac{1}{q}}+\left(\frac{k \mu \mu^{\frac{\alpha p+k}{k}}}{\alpha p+k}\right)^{\frac{1}{p}}\right. \\
& \left.\quad \times\left(\sigma_{3}\left|\Upsilon^{\prime}\left(b_{2}\right)\right|^{q}+\sigma_{4}\left|\Upsilon^{\prime}\left(b_{1}\right)\right|^{q}\right)^{\frac{1}{q}}\right],
\end{aligned}
$$

where $\sigma_{1}, \sigma_{2}, \sigma_{3}$ and $\sigma_{4}$ are already defined in Theorem 2.7.

Theorem 2.8 Let $\Upsilon:\left[b_{1}, b_{2}\right] \rightarrow \mathbb{R}$ be a function on $\left(b_{1}, b_{2}\right)$ with $b_{1}<b_{2}$ and $\left|\Upsilon^{\prime}\right|^{q}$ be an harmonic convex function with $q \geq 1$ and $\lambda, \mu \in[0, \infty)$ with $\lambda+\mu \neq 0$, then

$$
\begin{aligned}
& \mid \frac{\Omega(\lambda) \Upsilon\left(b_{2}\right)+\Omega(\mu) \Upsilon\left(b_{1}\right)}{\lambda+\mu} \\
& \quad-\frac{1}{\lambda+\mu}\left[\left(\frac{1}{\left.b_{2}\right)^{+}} I_{\Phi} \Upsilon \circ \Psi\left(\frac{\lambda b_{2}+\mu b_{1}}{b_{1} b_{2}(\lambda+\mu)}\right)+{ }_{\left(\frac{1}{b_{1}}\right)} I_{\Phi} \Upsilon \circ \Psi\left(\frac{\lambda b_{2}+\mu b_{1}}{b_{1} b_{2}(\lambda+\mu)}\right)\right] \mid\right. \\
& \leq b_{1} b_{2}\left(b_{2}-b_{1}\right)\left[\left(\int_{0}^{\lambda} \frac{\Omega(\tau)}{\left((\lambda-\tau) b_{2}+(\mu+\tau) b_{1}\right)^{2}} \mathrm{~d} \tau\right)^{1-\frac{1}{q}}\right. \\
& \quad \times\left(\int_{0}^{\lambda} \frac{\Omega(\tau)}{\left((\lambda-\tau) b_{2}+(\mu+\tau) b_{1}\right)^{2}}\left(\frac{\mu+\tau}{\lambda+\mu}\left|\Upsilon^{\prime}\left(b_{2}\right)\right|^{q}+\frac{\lambda-\tau}{\lambda+\mu}\left|\Upsilon^{\prime}\left(b_{1}\right)\right|^{q}\right) \mathrm{d} \tau\right)^{\frac{1}{q}} \\
& \quad+\left(\int_{0}^{\mu} \frac{\Omega(\tau)}{\left((\lambda+\tau) b_{2}+(\mu-\tau) b_{1}\right)^{2}} \mathrm{~d} \tau\right)^{1-\frac{1}{q}} \\
& \left.\quad \times\left(\int_{0}^{\mu} \frac{\Omega(\tau)}{\left((\lambda+\tau) b_{2}+(\mu-\tau) b_{1}\right)^{2}}\left(\frac{\mu-\tau}{\lambda+\mu}\left|\Upsilon^{\prime}\left(b_{2}\right)\right|^{q}+\frac{\lambda+\tau}{\lambda+\mu}\left|\Upsilon^{\prime}\left(b_{1}\right)\right|^{q}\right) \mathrm{d} \tau\right)^{\frac{1}{q}}\right] .
\end{aligned}
$$

Proof Using Lemma 2.3, the modulus property, the power mean inequality and the harmonic convexity of of $\left|\Upsilon^{\prime}\right|^{q}$, we have

$$
\begin{aligned}
& \mid \frac{\Omega(\lambda) \Upsilon\left(b_{2}\right)+\Omega(\mu) \Upsilon\left(b_{1}\right)}{\lambda+\mu} \\
& \quad-\frac{1}{\lambda+\mu}\left[\left(\frac{1}{\left.b_{2}\right)^{+}} I_{\Phi} \Upsilon \circ \Psi\left(\frac{\lambda b_{2}+\mu b_{1}}{b_{1} b_{2}(\lambda+\mu)}\right)+{ }_{\left(\frac{1}{b_{1}}\right)^{-}} I_{\Phi} \Upsilon \circ \Psi\left(\frac{\lambda b_{2}+\mu b_{1}}{b_{1} b_{2}(\lambda+\mu)}\right)\right] \mid\right. \\
& \leq b_{1} b_{2}\left(b_{2}-b_{1}\right)\left[\int_{0}^{\lambda} \frac{\Omega(\tau)}{\left((\lambda-\tau) b_{2}+(\mu+\tau) b_{1}\right)^{2}}\left|\Upsilon^{\prime}\left(\frac{b_{1} b_{2}(\lambda+\mu)}{(\lambda-\tau) b_{2}+(\mu+\tau) b_{1}}\right)\right| \mathrm{d} \tau\right. \\
& \left.\quad+\int_{0}^{\mu} \frac{\Omega(\tau)}{\left((\lambda+\tau) b_{2}+(\mu-\tau) b_{1}\right)^{2}}\left|\Upsilon^{\prime}\left(\frac{b_{1} b_{2}(\lambda+\mu)}{(\lambda+\tau) b_{2}+(\mu-\tau) b_{1}}\right)\right| \mathrm{d} \tau\right] \\
& \leq b_{1} b_{2}\left(b_{2}-b_{1}\right)\left[\left(\int_{0}^{\lambda} \frac{\Omega(\tau)}{\left((\lambda-\tau) b_{2}+(\mu+\tau) b_{1}\right)^{2}} \mathrm{~d} \tau\right)^{1-\frac{1}{q}}\right. \\
& \quad \times\left(\int_{0}^{\lambda} \frac{\Omega(\tau)}{\left((\lambda-\tau) b_{2}+(\mu+\tau) b_{1}\right)^{2}}\right.
\end{aligned}
$$




$$
\begin{aligned}
& \left.\times\left|\Upsilon^{\prime}\left(\frac{b_{1} b_{2}(\lambda+\mu)}{(\lambda-\tau) b_{2}+(\mu+\tau) b_{1}}\right)\right| \mathrm{d} \tau\right)^{\frac{1}{q}} \\
& +\left(\int_{0}^{\mu} \frac{\Omega(\tau)}{\left((\lambda+\tau) b_{2}+(\mu-\tau) b_{1}\right)^{2}} \mathrm{~d} \tau\right)^{1-\frac{1}{q}} \\
& \left.\times\left(\int_{0}^{\mu} \frac{\Omega(\tau)}{\left((\lambda+\tau) b_{2}+(\mu-\tau) b_{1}\right)^{2}}\left|\Upsilon^{\prime}\left(\frac{b_{1} b_{2}(\lambda+\mu)}{(\lambda+\tau) b_{2}+(\mu-\tau) b_{1}}\right)\right| \mathrm{d} \tau\right)^{\frac{1}{q}}\right] \\
& \leq b_{1} b_{2}\left(b_{2}-b_{1}\right)\left[\left(\int_{0}^{\lambda} \frac{\Omega(\tau)}{\left((\lambda-\tau) b_{2}+(\mu+\tau) b_{1}\right)^{2}} \mathrm{~d} \tau\right)^{1-\frac{1}{q}}\right. \\
& \times\left(\int_{0}^{\lambda} \frac{\Omega(\tau)}{\left((\lambda-\tau) b_{2}+(\mu+\tau) b_{1}\right)^{2}}\right. \\
& \left.\times\left(\frac{\mu+\tau}{\lambda+\mu}\left|\Upsilon^{\prime}\left(b_{2}\right)\right|^{q}+\frac{\lambda-\tau}{\lambda+\mu}\left|\Upsilon^{\prime}\left(b_{1}\right)\right|^{q}\right) \mathrm{d} \tau\right)^{\frac{1}{q}} \\
& +\left(\int_{0}^{\mu} \frac{\Omega(\tau)}{\left((\lambda+\tau) b_{2}+(\mu-\tau) b_{1}\right)^{2}} \mathrm{~d} \tau\right)^{1-\frac{1}{q}} \\
& \left.\times\left(\int_{0}^{\mu} \frac{\Omega(\tau)}{\left((\lambda+\tau) b_{2}+(\mu-\tau) b_{1}\right)^{2}}\left(\frac{\mu-\tau}{\lambda+\mu}\left|\Upsilon^{\prime}\left(b_{2}\right)\right|^{q}+\frac{\lambda+\tau}{\lambda+\mu}\left|\Upsilon^{\prime}\left(b_{1}\right)\right|^{q}\right) \mathrm{d} \tau\right)^{\frac{1}{q}}\right] .
\end{aligned}
$$

This completes the proof.

Corollary 2.22 Choosing $\Phi(\tau)=\tau$ and $\lambda=\mu=1$ in Theorem 2.8, we have

$$
\begin{aligned}
& \left|\frac{\Upsilon\left(b_{2}\right)+\Upsilon\left(b_{1}\right)}{2}-\frac{b_{1} b_{2}}{b_{2}-b_{1}} \int_{\frac{1}{b_{2}}}^{\frac{1}{b_{1}}} \Upsilon \circ \Psi(x) \mathrm{d} x\right| \\
& \leq b_{1} b_{2}\left(b_{2}-b_{1}\right)\left[\sigma_{5}^{1-\frac{1}{q}}\left(\sigma_{6}\left|\Upsilon^{\prime}\left(b_{2}\right)\right|^{q}+\sigma_{7}\left|\Upsilon^{\prime}\left(b_{1}\right)\right|^{q}\right)^{\frac{1}{q}}\right. \\
& \left.\quad+\sigma_{8}^{1-\frac{1}{q}}\left(\sigma_{9}\left|\Upsilon^{\prime}\left(b_{2}\right)\right|^{q}+\sigma_{10}\left|\Upsilon^{\prime}\left(b_{1}\right)\right|^{q}\right)^{\frac{1}{q}}\right],
\end{aligned}
$$

where

$$
\begin{aligned}
& \sigma_{5}:=\frac{\left(b_{1}+b_{2}\right)^{-2}}{2}{ }_{2} F_{1}\left(2,2,3, \frac{b_{2}-b_{1}}{b_{1}+b_{2}}\right), \\
& \sigma_{6}:=\frac{\left(b_{1}+b_{2}\right)^{-2}}{4}{ }_{2} F_{1}\left(2,2,3, \frac{b_{2}-b_{1}}{b_{1}+b_{2}}\right)+\frac{\left(b_{1}+b_{2}\right)^{-2}}{12}{ }_{2} F_{1}\left(2,3,4, \frac{b_{2}-b_{1}}{b_{1}+b_{2}}\right), \\
& \sigma_{7}:=\frac{\left(b_{1}+b_{2}\right)^{-2}}{12}{ }_{2} F_{1}\left(2,2,4, \frac{b_{2}-b_{1}}{b_{1}+b_{2}}\right), \\
& \sigma_{8}:=\frac{\left(b_{1}+b_{2}\right)^{-2}}{2}{ }_{2} F_{1}\left(2,2,3, \frac{b_{1}-b_{2}}{b_{1}+b_{2}}\right), \\
& \sigma_{9}:=\frac{\left(b_{1}+b_{2}\right)^{-2}}{12}{ }_{2} F_{1}\left(2,2,4, \frac{b_{1}-b_{2}}{b_{1}+b_{2}}\right), \\
& \sigma_{10}:=\frac{\left(b_{1}+b_{2}\right)^{-2}}{4}{ }_{2} F_{1}\left(2,2,3, \frac{b_{1}-b_{2}}{b_{1}+b_{2}}\right)+\frac{\left(b_{1}+b_{2}\right)^{-2}}{12}{ }_{2} F_{1}\left(2,3,4, \frac{b_{1}-b_{2}}{b_{1}+b_{2}}\right) .
\end{aligned}
$$


Corollary 2.23 Taking $\Phi(\tau)=\frac{\tau^{\alpha}}{\Gamma(\alpha)}$ and $\lambda=\mu=1$ in Theorem 2.8, we obtain

$$
\begin{aligned}
& \mid \frac{\Upsilon\left(b_{2}\right)+\Upsilon\left(b_{1}\right)}{2}-\frac{\left(b_{1} b_{2}\right)^{\alpha} 2^{\alpha-1} \Gamma(\alpha+1)}{\left(b_{2}-b_{1}\right)^{\alpha}}\left[J_{\left(\frac{1}{b_{2}}\right)^{+}}^{\alpha} \Upsilon \circ \Psi\left(\frac{b_{1}+b_{2}}{2 b_{1} b_{2}}\right)\right. \\
& \left.\quad+J_{\left(\frac{1}{b_{1}}\right)^{-}}^{\alpha} \Upsilon \odot \Psi\left(\frac{b_{1}+b_{2}}{2 b_{1} b_{2}}\right)\right] \mid \\
& \leq b_{1} b_{2}\left(b_{2}-b_{1}\right)\left[\sigma_{11}^{1-\frac{1}{q}}\left(\sigma_{12}\left|\Upsilon^{\prime}\left(b_{2}\right)\right|^{q}+\sigma_{13}\left|\Upsilon^{\prime}\left(b_{1}\right)\right|^{q}\right)^{\frac{1}{q}}\right. \\
& \left.\quad+\sigma_{14}^{1-\frac{1}{q}}\left(\sigma_{15}\left|\Upsilon^{\prime}\left(b_{2}\right)\right|^{q}+\sigma_{16}\left|\Upsilon^{\prime}\left(b_{1}\right)\right|^{q}\right)^{\frac{1}{q}}\right],
\end{aligned}
$$

where

$$
\begin{aligned}
\sigma_{11}:= & \frac{\left(b_{1}+b_{2}\right)^{-2}}{\alpha+1}{ }_{2} F_{1}\left(2, \alpha+1, \alpha+2, \frac{b_{2}-b_{1}}{b_{1}+b_{2}}\right), \\
\sigma_{12}:= & \frac{\left(b_{1}+b_{2}\right)^{-2}}{2(\alpha+1)}{ }_{2} F_{1}\left(2, \alpha+1, \alpha+2, \frac{b_{2}-b_{1}}{b_{1}+b_{2}}\right) \\
& +\frac{\left(b_{1}+b_{2}\right)^{-2}}{2(\alpha+1)(\alpha+2)}{ }_{2} F_{1}\left(2, \alpha+2, \alpha+3, \frac{b_{2}-b_{1}}{b_{1}+b_{2}}\right), \\
\sigma_{13}:= & \frac{\left(b_{1}+b_{2}\right)^{-2}}{2(\alpha+1)(\alpha+2)}{ }_{2} F_{1}\left(2, \alpha+1, \alpha+3, \frac{b_{2}-b_{1}}{b_{1}+b_{2}}\right), \\
\sigma_{14}:= & \frac{\left(b_{1}+b_{2}\right)^{-2}}{\alpha+1}{ }_{2} F_{1}\left(2, \alpha+1, \alpha+2, \frac{b_{1}-b_{2}}{b_{1}+b_{2}}\right), \\
\sigma_{15}:= & \frac{\left(b_{1}+b_{2}\right)^{-2}}{2(\alpha+1)(\alpha+2)}{ }_{2} F_{1}\left(2, \alpha+1, \alpha+3, \frac{b_{1}-b_{2}}{b_{1}+b_{2}}\right), \\
\sigma_{16}:= & \frac{\left(b_{1}+b_{2}\right)^{-2}}{2(\alpha+1)}{ }_{2} F_{1}\left(2, \alpha+1, \alpha+2, \frac{b_{1}-b_{2}}{b_{1}+b_{2}}\right) \\
& +\frac{\left(b_{1}+b_{2}\right)^{-2}}{2(\alpha+1)(\alpha+2)}{ }_{2} F_{1}\left(2, \alpha+2, \alpha+3, \frac{b_{1}-b_{2}}{b_{1}+b_{2}}\right) .
\end{aligned}
$$

Corollary 2.24 Choosing $\Phi(\tau)=\frac{\tau^{\frac{\alpha}{k}}}{k \Gamma_{k}(\alpha)}$ and $\lambda=\mu=1$ in Theorem 2.8, we obtain

$$
\begin{aligned}
& \mid \frac{\Upsilon\left(b_{2}\right)+\Upsilon\left(b_{1}\right)}{2}-\frac{\left(b_{1} b_{2}\right)^{\frac{\alpha}{k}} 2^{\frac{\alpha}{k}-1} \Gamma_{k}(\alpha+k)}{\left(b_{2}-b_{1}\right)^{\frac{\alpha}{k}}}\left[{ }_{k} J_{\left(\frac{1}{b_{2}}\right)^{+}}^{\alpha} \Upsilon \circ \Psi\left(\frac{b_{1}+b_{2}}{2 b_{1} b_{2}}\right)\right. \\
& \left.\quad+{ }_{k} J_{\left(\frac{1}{b_{1}}\right)^{-}}^{\alpha} \bigcirc \Psi\left(\frac{b_{1}+b_{2}}{2 b_{1} b_{2}}\right)\right] \mid \\
& \leq b_{1} b_{2}\left(b_{2}-b_{1}\right)\left[\sigma_{16}^{\frac{1}{p}}\left(\sigma_{17}\left|\Upsilon^{\prime}\left(b_{2}\right)\right|^{q}+\sigma_{18}\left|\Upsilon^{\prime}\left(b_{1}\right)\right|^{q}\right)^{\frac{1}{q}}\right. \\
& \left.\quad+\sigma_{19}^{\frac{1}{p}}\left(\sigma_{20}\left|\Upsilon^{\prime}\left(b_{2}\right)\right|^{q}+\sigma_{21}\left|\Upsilon^{\prime}\left(b_{1}\right)\right|^{q}\right)^{\frac{1}{q}}\right],
\end{aligned}
$$

where

$$
\sigma_{16}:=\frac{k\left(b_{1}+b_{2}\right)^{-2}}{\alpha+k}{ }_{2} F_{1}\left(2, \alpha+k, \alpha+2 k, \frac{b_{2}-b_{1}}{b_{1}+b_{2}}\right)
$$




$$
\begin{aligned}
\sigma_{17}:= & \frac{k\left(b_{1}+b_{2}\right)^{-2}}{2(\alpha k)}{ }_{2} F_{1}\left(2, \alpha+k, \alpha+2 k, \frac{b_{2}-b_{1}}{b_{1}+b_{2}}\right) \\
& +\frac{k\left(b_{1}+b_{2}\right)^{-2}}{2(\alpha+k)(\alpha+2 k)}{ }_{2} F_{1}\left(2, \alpha+2 k, \alpha+3 k, \frac{b_{2}-b_{1}}{b_{1}+b_{2}}\right), \\
\sigma_{18}:= & \frac{k\left(b_{1}+b_{2}\right)^{-2}}{2(\alpha+k)(\alpha+2 k)}{ }_{2} F_{1}\left(2, \alpha+k, \alpha+3 k, \frac{b_{2}-b_{1}}{b_{1}+b_{2}}\right), \\
\sigma_{19}:= & \frac{k\left(b_{1}+b_{2}\right)^{-2}}{\alpha+k}{ }_{2} F_{1}\left(2, \alpha+k, \alpha+2 k, \frac{b_{1}-b_{2}}{b_{1}+b_{2}}\right), \\
\sigma_{20}:= & \frac{k\left(b_{1}+b_{2}\right)^{-2}}{2(\alpha+k)(\alpha+2 k)}{ }_{2} F_{1}\left(2, \alpha+k, \alpha+3 k, \frac{b_{1}-b_{2}}{b_{1}+b_{2}}\right), \\
\sigma_{21}:= & \frac{k\left(b_{1}+b_{2}\right)^{-2}}{2(\alpha+k)}{ }_{2} F_{1}\left(2, \alpha+k, \alpha+2 k, \frac{b_{1}-b_{2}}{b_{1}+b_{2}}\right) \\
& +\frac{k\left(b_{1}+b_{2}\right)^{-2}}{2(\alpha+k)(\alpha+2 k)}{ }_{2} F_{1}\left(2, \alpha+2 k, \alpha+3 k, \frac{b_{1}-b_{2}}{b_{1}+b_{2}}\right) .
\end{aligned}
$$

Theorem 2.9 Let $\Upsilon:\left[b_{1}, b_{2}\right] \subset(0,+\infty) \rightarrow \mathbb{R}$ be a differentiable function on $\left(b_{1}, b_{2}\right)$ with $b_{1}<b_{2}$. If $\left|\Upsilon^{\prime}\right|^{q}$ is an harmonic convex function with $q>1$ and $\frac{1}{p}+\frac{1}{q}=1$, then

$$
\begin{aligned}
& \left|\Upsilon\left(\frac{2 b_{1} b_{2}}{b_{1}+b_{2}}\right)-\frac{1}{2 \Delta(1)}\left[{\frac{1}{b_{2}}}^{+} I_{\Phi} \Upsilon \circ \Psi\left(\frac{1}{b_{1}}\right)+{ }_{b_{1}}-I_{\Phi} \Upsilon \circ \Psi\left(\frac{1}{b_{2}}\right)\right]\right| \\
& \leq \frac{b_{1} b_{2}\left(b_{2}-b_{1}\right)}{2 \Delta(1)}\left(\left(\int_{0}^{\frac{1}{2}}|\Delta(\tau)|^{p} \mathrm{~d} \tau\right)^{\frac{1}{p}}+\left(\int_{\frac{1}{2}}^{1}|\delta(\tau)|^{p} \mathrm{~d} \tau\right)^{\frac{1}{p}}\right) \\
& \quad \times\left(\left(N_{1}\left|\Upsilon^{\prime}\left(b_{1}\right)\right|^{q}+N_{2}\left|\Upsilon^{\prime}\left(b_{2}\right)\right|^{q}\right)^{\frac{1}{q}}+\left(N_{3}\left|\Upsilon^{\prime}\left(b_{1}\right)\right|^{q}+N_{4}\left|\Upsilon^{\prime}\left(b_{2}\right)\right|^{q}\right)^{\frac{1}{q}}\right),
\end{aligned}
$$

where

$$
\begin{aligned}
N_{1} & :=\int_{0}^{\frac{1}{2}} \frac{(1-\tau)}{\left(\tau b_{1}+(1-\tau) b_{2}\right)^{2 q}} \mathrm{~d} \tau \\
& =\frac{2^{2-2 q} b_{2}^{2-2 q}-\left(b_{1}+b_{2}\right)^{2-2 q}}{\left(b_{2}-b_{1}\right)^{2}(2-2 q) 2^{2-2 q}}-b_{1} \frac{2^{1-2 q} b_{2}{ }^{1-2 q}-\left(b_{1}+b_{2}\right)^{1-2 q}}{\left(b_{2}-b_{1}\right)^{2}(1-2 q) 2^{1-2 q}} \\
N_{2} & :=\int_{0}^{\frac{1}{2}} \frac{\tau}{\left(\tau b_{1}+(1-\tau) b_{2}\right)^{2 q}} \mathrm{~d} \tau \\
& =b_{2} \frac{2^{1-2 q} b_{2}{ }^{1-2 q}-\left(b_{1}+b_{2}\right)^{1-2 q}}{\left(b_{2}-b_{1}\right)^{2}(1-2 q) 2^{1-2 q}}-\frac{2^{2-2 q} b_{2}{ }^{2-2 q}-\left(b_{1}+b_{2}\right)^{2-2 q}}{\left(b_{2}-b_{1}\right)^{2}(2-2 q) 2^{2-2 q}}, \\
N_{3} & :=\int_{0}^{\frac{1}{2}} \frac{\tau}{\left((1-\tau) \tau b_{1}+\tau b_{2}\right)^{2 q}} \mathrm{~d} \tau \\
& =\frac{\left(b_{1}+b_{2}\right)^{2-2 q}-2^{2-2 q} b_{1}{ }^{2-2 q}}{\left(b_{2}-b_{1}\right)^{2}(2-2 q) 2^{2-2 q}}-b_{1} \frac{\left(b_{1}+b_{2}\right)^{1-2 q}-2^{1-2 q} b_{1}{ }^{1-2 q}}{\left(b_{2}-b_{1}\right)^{2}(1-2 q) 2^{1-2 q}}, \\
N_{4} & :=\int_{0}^{\frac{1}{2}} \frac{1-\tau}{\left((1-\tau) \tau b_{1}+\tau b_{2}\right)^{2 q}} \mathrm{~d} \tau \\
& =b_{2} \frac{\left(b_{1}+b_{2}\right)^{1-2 q}-2^{1-2 q} b_{1}{ }^{1-2 q}}{\left(b_{2}-b_{1}\right)^{2}(1-2 q) 2^{1-2 q}}-\frac{\left(b_{1}+b_{2}\right)^{2-2 q}-2^{2-2 q} b_{1}{ }^{2-2 q}}{\left(b_{2}-b_{1}\right)^{2}(2-2 q) 2^{2-2 q}},
\end{aligned}
$$




$$
\begin{aligned}
N_{5} & :=\int_{\frac{1}{2}}^{1} \frac{1-\tau}{\left(\tau b_{1}+(1-\tau) b_{2}\right)^{2 q}} \mathrm{~d} \tau \\
& =\frac{\left(b_{1}+b_{2}\right)^{2-2 q}-2^{2-2 q} b_{1}{ }^{2-2 q}}{\left(b_{2}-b_{1}\right)^{2}(2-2 q) 2^{2-2 q}}-b_{1} \frac{\left(b_{1}+b_{2}\right)^{1-2 q}-2^{1-2 q} b_{1}{ }^{1-2 q}}{\left(b_{2}-b_{1}\right)^{2}(1-2 q) 2^{1-2 q}}, \\
N_{6} & :=\int_{\frac{1}{2}}^{1} \frac{\tau}{\left(\tau b_{1}+(1-\tau) b_{2}\right)^{2 q}} \mathrm{~d} \tau \\
& =b_{2} \frac{\left(b_{1}+b_{2}\right)^{1-2 q}-2^{1-2 q} b_{1}{ }^{1-2 q}}{\left(b_{2}-b_{1}\right)^{2}(1-2 q) 2^{1-2 q}}-\frac{\left(b_{1}+b_{2}\right)^{2-2 q}-2^{2-2 q} b_{1}{ }^{2-2 q}}{\left(b_{2}-b_{1}\right)^{2}(2-2 q) 2^{2-2 q}}, \\
N_{7} & :=\int_{\frac{1}{2}}^{1} \frac{\tau}{\left((1-\tau) b_{1}+\tau b_{2}\right)^{2 q}} \mathrm{~d} \tau \\
& =\frac{2^{2-2 q} b_{2}{ }^{2-2 q}-\left(b_{1}+b_{2}\right)^{2-2 q}}{\left(b_{2}-b_{1}\right)^{2}(2-2 q) 2^{2-2 q}}-b_{1} \frac{2^{1-2 q} b_{2}{ }^{1-2 q}-\left(b_{1}+b_{2}\right)^{1-2 q}}{\left(b_{2}-b_{1}\right)^{2}(1-2 q) 2^{1-2 q}}, \\
N_{8} & :=\int_{\frac{1}{2}}^{1} \frac{1-\tau}{\left((1-\tau) b_{1}+\tau b_{2}\right)^{2 q}} \mathrm{~d} \tau \\
& =b_{2} \frac{2^{1-2 q} b_{2}{ }^{1-2 q}-\left(b_{1}+b_{2}\right)^{1-2 q}}{\left(b_{2}-b_{1}\right)^{2}(1-2 q) 2^{1-2 q}}-\frac{2^{2-2 q} b_{2}{ }^{2-2 q}-\left(b_{1}+b_{2}\right)^{2-2 q}}{\left(b_{2}-b_{1}\right)^{2}(2-2 q) 2^{2-2 q}} .
\end{aligned}
$$

Also, it is easy to verify that $N_{1}=N_{7}, N_{2}=N_{8}, N_{3}=N_{5}$ and $N_{4}=N_{6}$.

Proof By using Lemma 2.4, the property of modulus, Hölder's inequality and the harmonic convexity of $\left|\Upsilon^{\prime}\right|^{q}$, we obtain the desired result. We omit here the proof.

Corollary 2.25 Taking $\Phi(\tau)=\tau$ in Theorem 2.9, then

$$
\begin{aligned}
\mid \Upsilon & \left(\frac{2 b_{1} b_{2}}{b_{1}+b_{2}}\right)-\frac{b_{1} b_{2}}{b_{2}-b_{1}} \int_{b_{1}}^{b_{2}} \frac{\Upsilon(x)}{x^{2}} \mathrm{~d} x \mid \\
\leq & \frac{b_{1} b_{2}\left(b_{2}-b_{1}\right)}{2}\left(\frac{1}{2^{p+1}(p+1)}\right)^{\frac{1}{p}} \\
& \times\left(\left(N_{1}\left|\Upsilon^{\prime}\left(b_{1}\right)\right|^{q}+N_{2}\left|\Upsilon^{\prime}\left(b_{2}\right)\right|^{q}\right)^{\frac{1}{q}}+\left(N_{3}\left|\Upsilon^{\prime}\left(b_{1}\right)\right|^{q}+N_{4}\left|\Upsilon^{\prime}\left(b_{2}\right)\right|^{q}\right)^{\frac{1}{q}}\right) .
\end{aligned}
$$

Corollary 2.26 Choosing $\Phi(\tau)=\frac{\tau^{\alpha}}{\Gamma(\alpha)}$ in Theorem 2.9, then

$$
\begin{aligned}
& \left|\Upsilon\left(\frac{2 b_{1} b_{2}}{b_{1}+b_{2}}\right)-\frac{\Gamma(\alpha+1)}{2}\left(\frac{b_{1} b_{2}}{b_{2}-b_{1}}\right)^{\alpha}\left[J_{\frac{1}{b_{2}}}^{\alpha}+\Upsilon \circ \Psi\left(\frac{1}{b_{1}}\right)+J_{\frac{1}{b_{1}}}^{\alpha}-\Upsilon \circ \Psi\left(\frac{1}{b_{2}}\right)\right]\right| \\
& \leq \frac{b_{1} b_{2}\left(b_{2}-b_{1}\right)}{2}\left(\frac{\left(1+2^{\alpha p-1}(\alpha p-1)\right)}{2^{\alpha p}(\alpha p+1)}\right)^{\frac{1}{p}} \\
& \times\left(\left(N_{1}\left|\Upsilon^{\prime}\left(b_{1}\right)\right|^{q}+N_{2}\left|\Upsilon^{\prime}\left(b_{2}\right)\right|^{q}\right)^{\frac{1}{q}}+\left(N_{3}\left|\Upsilon^{\prime}\left(b_{1}\right)\right|^{q}+N_{4}\left|\Upsilon^{\prime}\left(b_{2}\right)\right|^{q}\right)^{\frac{1}{q}}\right) .
\end{aligned}
$$


Corollary 2.27 Taking $\Phi(\tau)=\frac{\frac{\alpha}{k}}{k \Gamma_{k}(\alpha)}$ in Theorem 2.9, then

$$
\begin{aligned}
\mid \Upsilon( & \left.\frac{2 b_{1} b_{2}}{b_{1}+b_{2}}\right)-\frac{\Gamma_{k}(\alpha+k)}{2}\left(\frac{b_{1} b_{2}}{b_{2}-b_{1}}\right)^{\frac{\alpha}{k}}\left[J_{\frac{1}{b_{2}}}^{\alpha}+\Upsilon \circ \Psi\left(\frac{1}{b_{1}}\right)+{ }_{k} J_{\frac{1}{b_{1}}}^{\alpha}-\Upsilon \circ \Psi\left(\frac{1}{b_{2}}\right)\right] \mid \\
\leq & \frac{b_{1} b_{2}\left(b_{2}-b_{1}\right)}{2}\left(\frac{\left(k+2^{\frac{\alpha p}{k}}-1(\alpha p-k)\right)}{2^{\frac{\alpha p}{k}}(\alpha p+k)}\right)^{\frac{1}{p}} \\
& \times\left(\left(N_{1}\left|\Upsilon^{\prime}\left(b_{1}\right)\right|^{q}+N_{2}\left|\Upsilon^{\prime}\left(b_{2}\right)\right|^{q}\right)^{\frac{1}{q}}+\left(N_{3}\left|\Upsilon^{\prime}\left(b_{1}\right)\right|^{q}+N_{4}\left|\Upsilon^{\prime}\left(b_{2}\right)\right|^{q}\right)^{\frac{1}{q}}\right) .
\end{aligned}
$$

Theorem 2.10 Let $\Upsilon:\left[b_{1}, b_{2}\right] \subset(0,+\infty) \rightarrow \mathbb{R}$ be a differentiable function on $\left(b_{1}, b_{2}\right)$ with $b_{1}<b_{2}$. If $\left|\Upsilon^{\prime}\right|^{q}$ is an harmonic convex function with $q \geq 1$, then

$$
\begin{aligned}
& \left|\Upsilon\left(\frac{2 b_{1} b_{2}}{b_{1}+b_{2}}\right)-\frac{1}{2 \Delta(1)}\left[{\frac{1}{b_{2}}}^{+} I_{\Phi} \Upsilon \circ \Psi\left(\frac{1}{b_{1}}\right)+{\frac{1}{b_{1}}}^{-} I_{\Phi} \Upsilon \circ \Psi\left(\frac{1}{b_{2}}\right)\right]\right| \\
& \leq \frac{b_{1} b_{2}\left(b_{2}-b_{1}\right)}{2 \Delta(1)} \\
& \times\left[( \int _ { 0 } ^ { \frac { 1 } { 2 } } | \Delta ( \tau ) | \mathrm { d } \tau ) ^ { 1 - \frac { 1 } { q } } \left\{\left(\int_{0}^{\frac{1}{2}} \frac{(1-\tau)|\Delta(\tau)|}{\left(\tau b_{1}+(1-\tau) b_{2}\right)^{2}} \mathrm{~d} \tau\left|\Upsilon^{\prime}\left(b_{1}\right)\right|^{q}\right.\right.\right. \\
& \left.+\int_{0}^{\frac{1}{2}} \frac{\tau|\Delta(\tau)|}{\left(\tau b_{1}+(1-\tau) b_{2}\right)^{2}} \mathrm{~d} \tau\left|\Upsilon^{\prime}\left(b_{2}\right)\right|^{q}\right)^{\frac{1}{q}} \\
& \left.+\left(\int_{0}^{\frac{1}{2}} \frac{\tau|\Delta(\tau)|}{\left((1-\tau) b_{1}+\tau b_{2}\right)^{2}} \mathrm{~d} \tau\left|\Upsilon^{\prime}\left(b_{1}\right)\right|^{q}+\int_{0}^{\frac{1}{2}} \frac{(1-\tau)|\Delta(\tau)|}{\left((1-\tau) b_{1}+\tau b_{2}\right)^{2}} \mathrm{~d} \tau\left|\Upsilon^{\prime}\left(b_{2}\right)\right|^{q}\right)^{\frac{1}{q}}\right\} \\
& +\left(\int_{\frac{1}{2}}^{1}|\delta(\tau)| \mathrm{d} \tau\right)^{1-\frac{1}{q}}\left\{\left(\int_{\frac{1}{2}}^{1} \frac{(1-\tau)|\delta(\tau)|}{\left(\tau b_{1}+(1-\tau) b_{2}\right)^{2}} \mathrm{~d} \tau\left|\Upsilon^{\prime}\left(b_{1}\right)\right|^{q}\right.\right. \\
& \left.+\int_{\frac{1}{2}}^{1} \frac{\tau|\delta(\tau)|}{\left(\tau b_{1}+(1-\tau) b_{2}\right)^{2}} \mathrm{~d} \tau\left|\Upsilon^{\prime}\left(b_{2}\right)\right|^{q}\right)^{\frac{1}{q}} \\
& +\left(\int_{\frac{1}{2}}^{1} \frac{\tau|\delta(\tau)|}{\left((1-\tau) b_{1}+\tau b_{2}\right)^{2}} \mathrm{~d} \tau\left|\Upsilon^{\prime}\left(b_{1}\right)\right|^{q}\right. \\
& \left.\left.\left.+\int_{\frac{1}{2}}^{1} \frac{(1-\tau)|\delta(\tau)|}{\left((1-\tau) b_{1}+\tau b_{2}\right)^{2}} \mathrm{~d} \tau\left|\Upsilon^{\prime}\left(b_{2}\right)\right|^{q}\right)^{\frac{1}{q}}\right\}\right] .
\end{aligned}
$$

Proof By using Lemma 2.4, the property of modulus, the power mean inequality and the convexity of $\left|\Upsilon^{\prime}\right|^{q}$ we obtain the desired result. We omit here the proof.

Corollary 2.28 Taking $\Phi(\tau)=\tau$ in Theorem 2.10, then

$$
\begin{aligned}
\mid \Upsilon( & \left.\frac{2 b_{1} b_{2}}{b_{1}+b_{2}}\right)-\frac{b_{1} b_{2}}{b_{2}-b_{1}} \int_{b_{1}}^{b_{2}} \frac{\Upsilon(x)}{x^{2}} \mathrm{~d} x \mid \\
\leq & \frac{b_{1} b_{2}\left(b_{2}-b_{1}\right)}{2}\left(\frac{1}{8}\right)^{1-\frac{1}{q}} \\
& \times\left[\left(M_{1}\left|\Upsilon^{\prime}\left(b_{1}\right)\right|^{q}+M_{2}\left|\Upsilon^{\prime}\left(b_{2}\right)\right|^{q}\right)^{\frac{1}{q}}+\left(M_{3}\left|\Upsilon^{\prime}\left(b_{1}\right)\right|^{q}+M_{4}\left|\Upsilon^{\prime}\left(b_{2}\right)\right|^{q}\right)^{\frac{1}{q}}\right. \\
& \left.+\left(M_{2}\left|\Upsilon^{\prime}\left(b_{1}\right)\right|^{q}+M_{1}\left|\Upsilon^{\prime}\left(b_{2}\right)\right|^{q}\right)^{\frac{1}{q}}+\left(M_{4}\left|\Upsilon^{\prime}\left(b_{1}\right)\right|^{q}+M_{3}\left|\Upsilon^{\prime}\left(b_{2}\right)\right|^{q}\right)^{\frac{1}{q}}\right]
\end{aligned}
$$


Awn et al. Journal of Inequalities and Applications

(2022) $2022: 3$

Page 27 of 34

where

$$
\begin{aligned}
& M_{1}:=\frac{b_{2}{ }^{-2}}{8}{ }_{2} F_{1}\left(2,2,3, \frac{b_{2}-b_{1}}{2 b_{2}}\right)-\frac{b_{2}{ }^{-2}}{24}{ }_{2} F_{1}\left(2,3,4, \frac{b_{2}-b_{1}}{2 b_{2}}\right), \\
& M_{2}:=\frac{b_{2}{ }^{-2}}{24}{ }_{2} F_{1}\left(2,3,4, \frac{b_{2}-b_{1}}{2 b_{2}}\right), \quad M_{3}:=\frac{b_{1}^{-2}}{24}{ }_{2} F_{1}\left(2,3,4, \frac{b_{1}-b_{2}}{2 b_{1}}\right), \\
& M_{4}:=\frac{b_{1}{ }^{-2}}{8}{ }_{2} F_{1}\left(2,2,3, \frac{b_{1}-b_{2}}{2 b_{1}}\right)-\frac{b_{1}{ }^{-2}}{24}{ }_{2} F_{1}\left(2,3,4, \frac{b_{1}-b_{2}}{2 b_{1}}\right) .
\end{aligned}
$$

Corollary 2.29 Choosing $\Phi(\tau)=\frac{\tau^{\alpha}}{\Gamma(\alpha)}$ in Theorem 2.10, then

$$
\begin{aligned}
\mid \Upsilon( & \left.\frac{2 b_{1} b_{2}}{b_{1}+b_{2}}\right)-\frac{\Gamma(\alpha+1)}{2}\left(\frac{b_{1} b_{2}}{b_{2}-b_{1}}\right)^{\alpha}\left[J_{\frac{1}{b_{2}}}^{\alpha}+\Upsilon \circ \Psi\left(\frac{1}{b_{1}}\right)+J_{\frac{1}{b_{1}}}^{\alpha}-\Upsilon \circ \Psi\left(\frac{1}{b_{2}}\right)\right] \mid \\
\leq & \frac{b_{1} b_{2}\left(b_{2}-b_{1}\right)}{2} \\
& \times\left[( \frac { 1 } { 2 ^ { \alpha + 1 } ( \alpha + 1 ) } ) ^ { 1 - \frac { 1 } { q } } \left\{\left(M_{5}\left|\Upsilon^{\prime}\left(b_{1}\right)\right|^{q}+M_{6}\left|\Upsilon^{\prime}\left(b_{2}\right)\right|^{q}\right)^{\frac{1}{q}}\right.\right. \\
& \left.+\left(M_{7}\left|\Upsilon^{\prime}\left(b_{1}\right)\right|^{q}+M_{8}\left|\Upsilon^{\prime}\left(b_{2}\right)\right|^{q}\right)^{\frac{1}{q}}\right\} \\
& +\left(\frac{2^{\alpha}(\alpha-1)+1}{2^{\alpha+1}(\alpha+1)}\right)^{1-\frac{1}{q}}\left\{\left(M_{6}\left|\Upsilon^{\prime}\left(b_{1}\right)\right|^{q}+M_{5}\left|\Upsilon^{\prime}\left(b_{2}\right)\right|^{q}\right)^{\frac{1}{q}}\right. \\
& \left.\left.+\left(M_{8}\left|\Upsilon^{\prime}\left(b_{1}\right)\right|^{q}+M_{7}\left|\Upsilon^{\prime}\left(b_{2}\right)\right|^{q}\right)^{\frac{1}{q}}\right\}\right],
\end{aligned}
$$

where

$$
\begin{aligned}
M_{5}:= & \frac{b_{2}{ }^{-2}}{2^{\alpha+1}(\alpha+1)}{ }_{2} F_{1}\left(2, \alpha+1, \alpha+2, \frac{b_{2}-b_{1}}{2 b_{2}}\right) \\
& -\frac{b_{2}{ }^{-2}}{2^{\alpha+2}(\alpha+2)}{ }_{2} F_{1}\left(2, \alpha+2, \alpha+3, \frac{b_{2}-b_{1}}{2 b_{2}}\right), \\
M_{6}:= & \frac{b_{2}{ }^{-2}}{2^{\alpha+2}(\alpha+2)}{ }_{2} F_{1}\left(2, \alpha+2, \alpha+3, \frac{b_{2}-b_{1}}{2 b_{2}}\right), \\
M_{7}:= & \frac{b_{1}{ }^{-2}}{2^{\alpha+2}(\alpha+2)}{ }_{2} F_{1}\left(2, \alpha+2, \alpha+3, \frac{b_{1}-b_{2}}{2 b_{1}}\right), \\
M_{8}:= & \frac{b_{1}{ }^{-2}}{2^{\alpha+1}(\alpha+1)}{ }_{2} F_{1}\left(2, \alpha+1, \alpha+2, \frac{b_{1}-b_{2}}{2 b_{1}}\right) \\
& -\frac{b_{1}{ }^{-2}}{2^{\alpha+2}(\alpha+2)}{ }_{2} F_{1}\left(2, \alpha+2, \alpha+3, \frac{b_{1}-b_{2}}{2 b_{1}}\right) .
\end{aligned}
$$

Corollary 2.30 Taking $\Phi(\tau)=\frac{\tau \frac{\alpha}{k}}{k \Gamma_{k}(\alpha)}$ in Theorem 2.10, then

$$
\begin{aligned}
& \left|\Upsilon\left(\frac{2 b_{1} b_{2}}{b_{1}+b_{2}}\right)-\frac{\Gamma_{k}(\alpha+k)}{2}\left(\frac{b_{1} b_{2}}{b_{2}-b_{1}}\right)^{\frac{\alpha}{k}}\left[J_{k}^{\alpha}+\Upsilon \circ \Psi\left(\frac{1}{b_{1}}\right)+{ }_{k} J_{\frac{1}{b_{1}}}^{\alpha}-\Upsilon \circ \Psi\left(\frac{1}{b_{2}}\right)\right]\right| \\
& \quad \times\left[( \frac { k } { 2 ^ { \frac { \alpha } { k } + 1 } ( \alpha + k ) } ) ^ { 1 - \frac { 1 } { q } } \left\{\left(M_{9}\left|\Upsilon^{\prime}\left(b_{1}\right)\right|^{q}+M_{10}\left|\Upsilon^{\prime}\left(b_{2}\right)\right|^{q}\right)^{\frac{1}{q}}\right.\right.
\end{aligned}
$$




$$
\begin{aligned}
& \left.+\left(M_{11}\left|\Upsilon^{\prime}\left(b_{1}\right)\right|^{q}+M_{12}\left|\Upsilon^{\prime}\left(b_{2}\right)\right|^{q}\right)^{\frac{1}{q}}\right\} \\
& +\left(\frac{2^{\frac{\alpha}{k}}(\alpha-k)+k}{2^{\frac{\alpha}{k}+1}(\alpha+k)}\right)^{1-\frac{1}{q}}\left\{\left(M_{10}\left|\Upsilon^{\prime}\left(b_{1}\right)\right|^{q}+M_{9}\left|\Upsilon^{\prime}\left(b_{2}\right)\right|^{q}\right)^{\frac{1}{q}}\right. \\
& \left.\left.+\left(M_{12}\left|\Upsilon^{\prime}\left(b_{1}\right)\right|^{q}+M_{11}\left|\Upsilon^{\prime}\left(b_{2}\right)\right|^{q}\right)^{\frac{1}{q}}\right\}\right]
\end{aligned}
$$

where

$$
\begin{aligned}
M_{9}:= & \frac{k b_{2}{ }^{-2}}{2^{\frac{\alpha}{k}+1}(\alpha+k)}{ }_{2} F_{1}\left(2, \alpha+k, \alpha+2 k, \frac{1}{k}\left(\frac{b_{2}-b_{1}}{2 b_{2}}\right)\right) \\
& -\frac{k b_{2}{ }^{-2}}{2^{\frac{\alpha}{k}+2}(\alpha+2 k)}{ }_{2} F_{1}\left(2, \alpha+2 k, \alpha+3 k, \frac{1}{k}\left(\frac{b_{2}-b_{1}}{2 b_{2}}\right)\right), \\
M_{10}:= & \frac{k b_{2}{ }^{-2}}{2^{\frac{\alpha}{k}+2}(\alpha+2 k)}{ }_{2} F_{1}\left(2, \alpha+2 k, \alpha+3 k, \frac{1}{k}\left(\frac{b_{2}-b_{1}}{2 b_{2}}\right)\right), \\
M_{11}:= & \frac{k b_{1}^{-2}}{2^{\frac{\alpha}{k}+2}(\alpha+2 k)}{ }_{2} F_{1}\left(2, \alpha+2 k, \alpha+3 k, \frac{1}{k}\left(\frac{b_{1}-b_{2}}{2 b_{1}}\right)\right), \\
M_{12}:= & \frac{k b_{1}{ }^{-2}}{2^{\frac{\alpha}{k}+1}(\alpha+k)}{ }_{2} F_{1}\left(2, \alpha+k, \alpha+2 k, \frac{1}{k}\left(\frac{b_{1}-b_{2}}{2 b_{1}}\right)\right) \\
& -\frac{k b_{1}^{-2}}{2^{\frac{\alpha}{k}+2}(\alpha+2 k)}{ }_{2} F_{1}\left(2, \alpha+2 k, \alpha+3 k, \frac{1}{k}\left(\frac{b_{1}-b_{2}}{2 b_{1}}\right)\right) .
\end{aligned}
$$

Remark For other suitable choices of function $\Phi$, several new interesting inequalities can be found from our results. We omit here their proofs and the details are left to the interested reader.

\section{Applications}

\subsection{Application to special means}

We shall consider the following special means for different positive real numbers $b_{1}$ and $b_{2}$, where $b_{1}<b_{2}$ :

- Arithmetic mean: $\mathcal{A}\left(b_{1}, b_{2}\right)=\frac{b_{1}+b_{2}}{2}$;

- Harmonic mean: $\mathcal{H}\left(b_{1}, b_{2}\right)=\frac{2 b_{1} b_{2}}{b_{1}+b_{2}}$;

- $r$-Logarithmic mean: $\mathcal{L}_{r}\left(b_{1}, b_{2}\right)=\left(\frac{b_{2}{ }^{r+1}-b_{1}{ }^{r+1}}{(r+1)\left(b_{2}-b_{1}\right)}\right)^{\frac{1}{r}}, r \in \mathbb{R} \backslash\{0,-1\}$.

Using our results, we are in a position to prove the following inequalities regarding the above special means.

Proposition 3.1 Let $r, b_{1}, b_{2} \in \mathbb{R}, 0<b_{1}<b_{2}$ with $r \geq 1, q>1$ and $\frac{1}{p}+\frac{1}{q}=1$, then

$$
\begin{aligned}
& \left|A\left(b_{1}{ }^{\frac{r}{q}+2}, b_{2}{ }^{\frac{r}{q}+2}\right)-\frac{q}{r+q} b_{1} b_{2} \mathcal{L}_{\frac{r}{q}}^{\frac{r}{q}}\left(b_{1}, b_{2}\right)\right| \\
& \quad \leq b_{1} b_{2}\left(b_{2}-b_{1}\right)\left(\frac{1}{p+1}\right)^{\frac{1}{p}}\left[\left(\sigma_{1}^{\star} b_{2}{ }^{r+q}+\sigma_{2}^{\star} b_{1}^{r+q}\right)^{\frac{1}{q}}+\left(\sigma_{3}^{\star} b_{2}{ }^{r+q}+\sigma_{4}^{\star} b_{1}{ }^{r+q}\right)^{\frac{1}{q}}\right]
\end{aligned}
$$


where

$$
\begin{aligned}
\sigma_{1}^{\star} & :=\int_{0}^{1} \frac{\left((1-\tau) b_{2}+(1+\tau) b_{1}\right)^{-2 q}(1+\tau)}{2} \mathrm{~d} \tau \\
& =\frac{\left(b_{2}+b_{1}\right)^{1-2 q}-2\left(2 b_{1}\right)^{1-2 q}}{2\left(b_{2}-b_{1}\right)(1-2 q)}-\frac{\left(2 b_{1}\right)^{2-2 q}-\left(b_{2}+b_{1}\right)^{2-2 q}}{2\left(b_{2}-b_{1}\right)^{2}(1-2 q)(2-2 q)}, \\
\sigma_{2}^{\star} & :=\int_{0}^{1} \frac{\left((1-\tau) b_{2}+(1+\tau) b_{1}\right)^{-2 q}(1-\tau)}{2} \mathrm{~d} \tau \\
& =\frac{\left(b_{2}+b_{1}\right)^{1-2 q}}{2\left(b_{2}-b_{1}\right)(1-2 q)}+\frac{\left(2 b_{1}\right)^{2-2 q}-\left(b_{1}+b_{2}\right)^{2-2 q}}{2\left(b_{2}-b_{1}\right)^{2}(1-2 q)(2-2 q)}, \\
\sigma_{3}^{\star} & :=\int_{0}^{1} \frac{\left((1+\tau) b_{2}+(1-\tau) b_{1}\right)^{-2 q}(1-\tau)}{2} \mathrm{~d} \tau \\
& =\frac{\left(2 b_{2}\right)^{2-2 q}-\left(b_{2}+b_{1}\right)^{2-2 q}}{2\left(b_{2}-b_{1}\right)^{2}(1-2 q)(2-2 q)}-\frac{\left(b_{2}+b_{1}\right)^{1-2 q}}{2\left(b_{2}-b_{1}\right)(1-2 q)}, \\
\sigma_{4}^{\star} & :=\int_{0}^{1} \frac{\left((1+\tau) b_{2}+(1-\tau) b_{1}\right)^{-2 q}(1+\tau)}{2} \mathrm{~d} \tau \\
& =\frac{\left(2 b_{2}\right)^{1-2 q}-\left(b_{2}+b_{1}\right)^{1-2 q}}{2\left(b_{2}-b_{1}\right)(1-2 q)}-\frac{\left(2 b_{2}\right)^{2-2 q}-\left(b_{2}+b_{1}\right)^{2-2 q}}{2\left(b_{2}-b_{1}\right)^{2}(1-2 q)(2-2 q)} .
\end{aligned}
$$

Proof Taking the harmonic convex function $\Upsilon(\tau)=\frac{q}{r+2 q} \tau^{\frac{r}{q}+2}$ for all $\tau>0$ in Corollary 2.19 and $\lambda=\mu=1$, we have the desired result.

Proposition 3.2 Let $r, b_{1}, b_{2} \in, 0<b_{1}<b_{2}$ with $r \geq 1, q>1$ and $\frac{1}{p}+\frac{1}{q}=1$, then

$$
\begin{aligned}
\left|\mathcal{H}^{\frac{r}{q}+2}\left(b_{1}, b_{2}\right)-\frac{q}{r+q} b_{1} b_{2} \mathcal{L}_{\frac{r}{q}}^{\frac{r}{q}}\left(b_{1}, b_{2}\right)\right| \\
\leq \frac{(r+2 q) b_{1} b_{2}\left(b_{2}-b_{1}\right)}{q}\left(\frac{1}{2^{p+2}(p+1)}\right)^{\frac{1}{p}} \\
\quad \times\left(\mathcal{A}^{\frac{1}{q}}\left(N_{1} b_{1}{ }^{r+q}, N_{2} b_{2}{ }^{r+q}\right)+\mathcal{A}^{\frac{1}{q}}\left(N_{3} b_{1}^{r+q}, N_{4} b_{2}{ }^{r+q}\right)\right) .
\end{aligned}
$$

Proof Choosing the harmonic convex function $\Upsilon(\tau)=\frac{q}{r+2 q} \tau^{\frac{r}{q}+2}$ for all $\tau>0$ in Corollary 2.25 , we obtain the desired result.

Proposition 3.3 Let $r, b_{1}, b_{2} \in \mathbb{R}, 0<b_{1}<b_{2}$ with $r, q \geq 1$, then

$$
\begin{aligned}
\left|\mathcal{H}^{\frac{r}{q}+2}\left(b_{1}, b_{2}\right)-\frac{q}{r+q} b_{1} b_{2} \mathcal{L}_{\frac{r}{q}}^{\frac{r}{q}}\left(b_{1}, b_{2}\right)\right| \\
\leq \\
\quad \frac{(r+2 q) b_{1} b_{2}\left(b_{2}-b_{1}\right)}{q}\left(\frac{1}{16}\right)^{1-\frac{1}{q}} \\
\quad \times\left[\mathcal{A}^{\frac{1}{q}}\left(M_{1} b_{1}{ }^{r+q}, M_{2} b_{2}{ }^{r+q}\right)+\mathcal{A}^{\frac{1}{q}}\left(M_{3} b_{1}{ }^{r+q}, M_{4} b_{2}{ }^{r+q}\right)\right. \\
\left.\quad+\mathcal{A}^{\frac{1}{q}}\left(M_{2} b_{1}{ }^{r+q}, M_{1} b_{2}{ }^{r+q}\right)+\mathcal{A}^{\frac{1}{q}}\left(M_{4} b_{1}{ }^{r+q}, M_{3} b_{2}{ }^{r+q}\right)\right] .
\end{aligned}
$$

Proof Taking the harmonic convex function $\Upsilon(\tau)=\frac{q}{r+2 q} \tau^{\frac{r}{q}+2}$ for all $\tau>0$ in Corollary 2.28, we obtain the desired result. 


\subsection{Application to error estimations}

Finally, let us consider some applications of the integral inequalities obtained above to find new error bounds for the following quadrature formulas. Let $\mathcal{P}: b_{1}=x_{0}<x_{1}<\cdots<x_{n-1}<$ $x_{n}=b_{2}$ be a partition of $\left[b_{1}, b_{2}\right] \subset(0,+\infty)$. We denote, respectively

$$
\begin{aligned}
& \mathcal{T}_{1}(\mathcal{P}, \Upsilon):=\sum_{i=0}^{n-1} \frac{\left(\Upsilon\left(x_{i}\right)+\Upsilon\left(x_{i+1}\right)\right)}{(m+1) x_{i} x_{i+1}} h_{i}, \quad \mathcal{T}_{2}(\mathcal{P}, \Upsilon):=\sum_{i=0}^{n-1} \frac{\Upsilon\left(\frac{2 x_{i} x_{i+1}}{x_{i}+x_{i+1}}\right)}{x_{i} x_{i+1}} h_{i} \\
& \int_{\frac{1}{b_{2}}}^{\frac{1}{b_{1}}} \Upsilon \circ \Psi(x) d x:=\mathcal{T}_{1}(\mathcal{P}, \Upsilon)+\mathcal{R}_{1}(\mathcal{P}, \Upsilon), \\
& \int_{b_{1}}^{b_{2}} \frac{\Upsilon(x)}{x^{2}} d x:=\mathcal{T}_{2}(\mathcal{P}, \Upsilon)+\mathcal{R}_{2}(\mathcal{P}, \Upsilon),
\end{aligned}
$$

where $m \in \mathbb{N}$, and $\mathcal{R}_{1}(\mathcal{P}, \Upsilon)$ and $\mathcal{R}_{2}(\mathcal{P}, \Upsilon)$ are the remainder terms and $h_{i}=x_{i+1}-x_{i}$ for $i=0,1,2, \ldots, n-1$. Using the above notations, we are in a position to prove the following error estimations.

Proposition 3.4 Let $\Upsilon:\left[b_{1}, b_{2}\right] \subset(0,+\infty) \rightarrow \mathbb{R}$ be a differentiable function on $\left(b_{1}, b_{2}\right)$ with $b_{1}<b_{2}$ and $m \in \mathbb{N}$. If $\left|\Upsilon^{\prime}\right|^{q}$ is an harmonic convex function with $q>1$ and $\frac{1}{p}+\frac{1}{q}=1$, then

$$
\begin{aligned}
& \left|\mathcal{R}_{1}(\mathcal{P}, \Upsilon)\right| \\
& \leq \sum_{i=0}^{n-1} h_{i}^{2}\left[\pi_{i, 1}^{\frac{1}{p}}\left(\frac{1}{(m+1)(q+1)(q+2)}\left|\Upsilon^{\prime}\left(x_{i}\right)\right|^{q}+\frac{m(q+2)+(q+1)}{(m+1)(q+1)(q+2)}\left|\Upsilon^{\prime}\left(x_{i+1}\right)\right|^{q}\right)^{\frac{1}{q}}\right. \\
& \left.\quad+\pi_{i, 2}^{\frac{1}{p}}\left(\frac{1}{(m+1)(q+1)(q+2)}\left|\Upsilon^{\prime}\left(x_{i+1}\right)\right|^{q}+\frac{m(q+2)+(q+1)}{(m+1)(q+1)(q+2)}\left|\Upsilon^{\prime}\left(x_{i}\right)\right|^{q}\right)^{\frac{1}{q}}\right]
\end{aligned}
$$

where

$$
\begin{aligned}
& \pi_{i, 1}:=\frac{\left(m x_{i}+x_{i+1}\right)^{1-2 p}}{h_{i}(1-2 p)}\left[1-\left(\frac{(m+1) x_{i}}{m x_{i}+x_{i+1}}\right)^{1-2 p}\right], \\
& \pi_{i, 2}:=\frac{\left(x_{i}+m x_{i+1}\right)^{1-2 p}}{h_{i}(1-2 p)}\left[\left(\frac{(m+1) x_{i+1}}{x_{i}+m x_{i+1}}\right)^{1-2 p}-1\right] .
\end{aligned}
$$

Proof By using Corollary 2.13 on the subintervals $\left[x_{i}, x_{i+1}\right](i=0,1,2, \ldots, n-1)$ of the partition $\mathcal{P}$ and summing the obtained inequality over $i$ from 0 to $n-1$, we have the desired result.

Proposition 3.5 Let $\Upsilon:\left[b_{1}, b_{2}\right] \subset(0,+\infty) \rightarrow \mathbb{R}$ be a differentiable function on $\left(b_{1}, b_{2}\right)$ with $b_{1}<b_{2}$ and $m \in \mathbb{N}$. If $\left|\Upsilon^{\prime}\right|^{q}$ is an harmonic convex function with $q \geq 1$, then

$$
\begin{aligned}
& \left|\mathcal{R}_{1}(\mathcal{P}, \Upsilon)\right| \\
& \leq \sum_{i=0}^{n-1} h_{i}^{2}\left[\pi_{i, 3}^{1-\frac{1}{q}}\left(\pi_{i, 4}\left|\Upsilon^{\prime}\left(x_{i}\right)\right|^{q}+\pi_{i, 5}\left|\Upsilon^{\prime}\left(x_{i+1}\right)\right|^{q}\right)^{\frac{1}{q}}\right. \\
& \left.\quad+\pi_{i, 6}^{1-\frac{1}{q}}\left(\pi_{i, 7}\left|\Upsilon^{\prime}\left(x_{i}\right)\right|^{q}+\pi_{i, 8}\left|\Upsilon^{\prime}\left(x_{i+1}\right)\right|^{q}\right)^{\frac{1}{q}}\right]
\end{aligned}
$$


where

$$
\begin{aligned}
\pi_{i, 3}:= & \frac{\left(m x_{i}+x_{i+1}\right)^{-2}}{2}{ }_{2} F_{1}\left(2,2,3, \frac{h_{i}}{m x_{i}+x_{i+1}}\right) \\
\pi_{i, 4}:= & \frac{\left(m x_{i}+x_{i+1}\right)^{-2}}{6(m+1)}{ }_{2} F_{1}\left(2,2,4, \frac{h_{i}}{m x_{i}+x_{i+1}}\right) \\
\pi_{i, 5}:= & \frac{m\left(m x_{i}+x_{i+1}\right)^{-2}}{2(m+1)}{ }_{2} F_{1}\left(2,2,3, \frac{h_{i}}{m x_{i}+x_{i+1}}\right) \\
& +\frac{\left(m x_{i}+x_{i+1}\right)^{-2}}{6(m+1)}{ }_{2} F_{1}\left(2,3,4, \frac{h_{i}}{m x_{i}+x_{i+1}}\right) \\
\pi_{i, 6}:= & \frac{\left(x_{i}+m x_{i+1}\right)^{-2}}{2}{ }_{2} F_{1}\left(2,2,3,-\frac{h_{i}}{x_{i}+m x_{i+1}}\right) \\
\pi_{i, 7}:= & \frac{m\left(x_{i}+m x_{i+1}\right)^{-2}}{2(m+1)}{ }_{2} F_{1}\left(2,2,3,-\frac{h_{i}}{x_{i}+m x_{i+1}}\right) \\
& +\frac{\left(x_{i}+m x_{i+1}\right)^{-2}}{6(m+1)}{ }_{2} F_{1}\left(2,3,4,-\frac{h_{i}}{x_{i}+m x_{i+1}}\right) \\
& \quad \frac{\left(x_{i}+m x_{i+1}\right)^{-2}}{6(m+1)}{ }_{2} F_{1}\left(2,2,4,-\frac{h_{i}}{x_{i}+m x_{i+1}}\right) .
\end{aligned}
$$

Proof By applying Corollary 2.16 on the subintervals $\left[x_{i}, x_{i+1}\right](i=0,1,2, \ldots, n-1)$ of the partition $\mathcal{P}$ and summing the obtained inequality over $i$ from 0 to $n-1$, we obtain the desired result.

Proposition 3.6 Let $\Upsilon:\left[b_{1}, b_{2}\right] \subset(0,+\infty) \rightarrow \mathbb{R}$ be a differentiable function on $\left(b_{1}, b_{2}\right)$ with $b_{1}<b_{2}$. If $\left|\Upsilon^{\prime}\right|^{q}$ is an harmonic convex function with $q>1$ and $\frac{1}{p}+\frac{1}{q}=1$, then

$$
\begin{aligned}
\left|\mathcal{R}_{2}(\mathcal{P}, \Upsilon)\right| & \\
\leq & \frac{1}{2}\left(\frac{1}{2^{p+1}(p+1)}\right)^{\frac{1}{p}} \\
& \times \sum_{i=0}^{n-1} h_{i}^{2}\left(\left(N_{i, 1}\left|\Upsilon^{\prime}\left(x_{i}\right)\right|^{q}+N_{i, 2}\left|\Upsilon^{\prime}\left(x_{i+1}\right)\right|^{q}\right)^{\frac{1}{q}}+\left(N_{i, 3}\left|\Upsilon^{\prime}\left(x_{i}\right)\right|^{q}+N_{i, 4}\left|\Upsilon^{\prime}\left(x_{i+1}\right)\right|^{q}\right)^{\frac{1}{q}}\right),
\end{aligned}
$$

where

$$
\begin{aligned}
& N_{i, 1}:=\frac{2^{2-2 q} x_{i+1}^{2-2 q}-\left(x_{i}+x_{i+1}\right)^{2-2 q}}{h_{i}^{2}(2-2 q) 2^{2-2 q}}-x_{i} \frac{2^{1-2 q} x_{i+1}^{1-2 q}-\left(x_{i}+x_{i+1}\right)^{1-2 q}}{h_{i}^{2}(1-2 q) 2^{1-2 q}}, \\
& N_{i, 2}:=x_{i+1} \frac{2^{1-2 q} x_{i+1}^{1-2 q}-\left(x_{i}+x_{i+1}\right)^{1-2 q}}{h_{i}^{2}(1-2 q) 2^{1-2 q}}-\frac{2^{2-2 q} x_{i+1}^{2-2 q}-\left(x_{i}+x_{i+1}\right)^{2-2 q}}{h_{i}^{2}(2-2 q) 2^{2-2 q}}, \\
& N_{i, 3}:=\frac{\left(x_{i}+x_{i+1}\right)^{2-2 q}-2^{2-2 q} x_{i}^{2-2 q}}{h_{i}^{2}(2-2 q) 2^{2-2 q}}-x_{i} \frac{\left(x_{i}+x_{i+1}\right)^{1-2 q}-2^{1-2 q} x_{i}^{1-2 q}}{h_{i}^{2}(1-2 q) 2^{1-2 q}} \\
& N_{i, 4}:=x_{i+1} \frac{\left(x_{i}+x_{i+1}\right)^{1-2 q}-2^{1-2 q} x_{i}^{1-2 q}}{h_{i}^{2}(1-2 q) 2^{1-2 q}}-\frac{\left(x_{i}+x_{i+1}\right)^{2-2 q}-2^{2-2 q} x_{i}^{2-2 q}}{h_{i}^{2}(2-2 q) 2^{2-2 q}} .
\end{aligned}
$$


Proof By using Corollary 2.25 on the subintervals $\left[x_{i}, x_{i+1}\right](i=0,1,2, \ldots, n-1)$ of the partition $\mathcal{P}$ and summing the obtained inequality over $i$ from 0 to $n-1$, we have the desired result.

Proposition 3.7 Let $\Upsilon:\left[b_{1}, b_{2}\right] \subset(0,+\infty) \rightarrow \mathbb{R}$ be a differentiable function on $\left(b_{1}, b_{2}\right)$ with $b_{1}<b_{2}$. If $\left|\Upsilon^{\prime}\right|^{q}$ is an harmonic convex function with $q \geq 1$, then

$$
\begin{aligned}
\left|\mathcal{R}_{2}(\mathcal{P}, \Upsilon)\right| & \\
\leq & \frac{1}{2}\left(\frac{1}{8}\right) \\
& \times \sum_{i=0}^{1-\frac{1}{q}} h_{i}^{2}\left[\left(M_{i, 1}\left|\Upsilon^{\prime}\left(x_{i}\right)\right|^{q}+M_{i, 2}\left|\Upsilon^{\prime}\left(x_{i+1}\right)\right|^{q}\right)^{\frac{1}{q}}+\left(M_{i, 3}\left|\Upsilon^{\prime}\left(x_{i}\right)\right|^{q}+M_{i, 4}\left|\Upsilon^{\prime}\left(x_{i+1}\right)\right|^{q}\right)^{\frac{1}{q}}\right. \\
& \left.+\left(M_{i, 2}\left|\Upsilon^{\prime}\left(x_{i}\right)\right|^{q}+M_{i, 1}\left|\Upsilon^{\prime}\left(x_{i+1}\right)\right|^{q}\right)^{\frac{1}{q}}+\left(M_{i, 4}\left|\Upsilon^{\prime}\left(x_{i}\right)\right|^{q}+M_{i, 3}\left|\Upsilon^{\prime}\left(x_{i+1}\right)\right|^{q}\right)^{\frac{1}{q}}\right],
\end{aligned}
$$

where

$$
\begin{aligned}
& M_{i, 1}:=\frac{x_{i+1}{ }^{-2}}{8}{ }_{2} F_{1}\left(2,2,3, \frac{h_{i}}{2 x_{i+1}}\right)-\frac{x_{i+1}{ }^{-2}}{24}{ }_{2} F_{1}\left(2,3,4, \frac{h_{i}}{2 x_{i+1}}\right), \\
& M_{i, 2}:=\frac{x_{i+1}{ }^{-2}}{24}{ }_{2} F_{1}\left(2,3,4, \frac{h_{i}}{2 x_{i+1}}\right), \quad M_{i, 3}:=\frac{x_{i}^{-2}}{24}{ }_{2} F_{1}\left(2,3,4,-\frac{h_{i}}{2 x_{i}}\right), \\
& M_{i, 4}:=\frac{x_{i}{ }^{-2}}{8}{ }_{2} F_{1}\left(2,2,3,-\frac{h_{i}}{2 x_{i}}\right)-\frac{x_{i}^{-2}}{24}{ }_{2} F_{1}\left(2,3,4,-\frac{h_{i}}{2 x_{i}}\right) .
\end{aligned}
$$

Proof By applying Corollary 2.28 on the subintervals $\left[x_{i}, x_{i+1}\right](i=0,1,2, \ldots, n-1)$ of the partition $\mathcal{P}$ and summing the obtained inequality over $i$ from 0 to $n-1$, we obtain the desired result.

Remark For suitable choices of function $\Upsilon$, we can obtain new inequalities using special means. Moreover, we can establish new bounds regarding error estimations of the quadrature formulas given above. We omit here their proofs and the details are left to the interested reader.

\section{Conclusion}

In this paper, we have derived some generalizations of fractional trapezium-like inequalities using the class of harmonic convex functions. Moreover, three new fractional integral identities are given and on applying them as auxiliary results some interesting integral inequalities are found. Our results unified many known ones, and they related some other unrelated results as well. Finally, some applications to special means for different positive real numbers and error estimations for quadrature formulas are obtained. This shows the efficiency of our results. To the best of our knowledge, these results are new in the literature and we believe that they will have a very deep impact in this field of inequalities, and also in pure and applied sciences. 


\section{Funding}

This research project is supported by the Thailand Science Research and Innovation (TSRI) Basic Research Fund: Fiscal year 2021 under project number 64A306000005.

Availability of data and materials

Not applicable.

\section{Declarations}

\section{Competing interests}

The authors declare that they have no competing interests.

\section{Authors' contributions}

All authors contributed equally to the writing of this paper. All authors read and approved the final manuscript.

\section{Author details}

'Department of Mathematics, Government College University, Faisalabad, Pakistan. ${ }^{2}$ Department of Mathematics, Faculty of Technical Science, University "Ismail Qemali", 9400, Vlora, Albania. ${ }^{3}$ Department of Mathematics, College of Arts and Sciences, Prince Sattam bin Abdulaziz University, Al-Kharj, Saudi Arabia. ${ }^{4}$ Center of Excellence in Theoretical and Computational Science (TaCS-CoE) \& KMUTT Fixed Point Research Laboratory, Room SCL 802 Fixed Point Laboratory, Science Laboratory Building, Department of Mathematics, King Mongkut's University of Technology Thonburi (KMUTT), 126 Pracha-Uthit Road, Bang Mod, Thung Khru, Bangkok 10140, Thailand. ${ }^{5}$ Department of Medical Research, China Medical University Hospital, China Medical University, Taichung 40402, Taiwan. ${ }^{6}$ NCAO Research Center, Fixed Point Theory and Applications Research Group, Center of Excellence in Theoretical and Computational Science (TaCS-CoE), Faculty of Science, King Mongkut's University of Technology Thonburi (KMUTT), 126 Pracha-Uthit Road, Bang Mod, Thung Khru, Bangkok 10140, Thailand.

\section{Publisher's Note}

Springer Nature remains neutral with regard to jurisdictional claims in published maps and institutional affiliations.

Received: 26 July 2021 Accepted: 9 December 2021 Published online: 03 January 2022

\section{References}

1. Hilfer, R.: In: Applications of Fractional Calculus in Physics, vol. 35, pp. 87-130. World Scientific, Singapore (2000)

2. Al-luhaibi, M.S.: An analytical treatment to fractional Fornberg-Whitham equation. Math. Sci. 11, 1-6 (2017)

3. Isscan, I.: Hermite-Hadamard type inequalities for harmonically convex functions. Hacet. J. Math. Stat. 43(6), 935-942 (2014)

4. Azemi, F.A., Calin, O.: Asian options with harmonic average. Appl. Math. Inf. Sci. 9(6), 1-9 (2015)

5. Noor, M.A.: Advanced Convex and Numerical Analysis, Lecture Notes. COMSATS Institute of Information Technology, Islamabad (2008-2016)

6. Sarikaya, M.Z., Set, E., Yaldiz, H., Basak, N.: Hermite-Hadamard's inequalities for fractional integrals and related fractional inequalities. Math. Comput. Model. 57, 2403-2407 (2013)

7. Işcan, I., Wu, S.H.: Hermite-Hadamard type inequalities for harmonically convex functions via fractional integrals. Appl. Math. Comput. 238, 237-244 (2014)

8. Awan, M.U., Talib, S., Chu, Y.M., Noor, M.A., Noor, K.I.: Some new refinements of Hermite-Hadamard-type inequalities involving $\psi_{k}-$ Riemann-Liouville fractional integrals and applications. Math. Probl. Eng. 2020, Article ID 3051920 (2020)

9. Dragomir, S.S., Pearce, C.E.M.: Selected Topics on Hermite-Hadamard Inequalities and Applications. Victoria University, Australia (2000)

10. Kilbas, A.A., Srivastava, H.M., Trujillo, J.J.: Theory and Applications of Fractional Differential Equations. North-Holland Mathematics Studies, vol. 204. Elsevier, Amsterdam (2006)

11. Mubeen, S., Habibullah, G.M.: k-fractional integrals and application. Int. J. Contemp. Math. Sci. 7(2), 89-94 (2012)

12. Katugampola, U.N.: New approach to a generalized fractional integral. Appl. Math. Comput. 218(3), 860-865 (2011)

13. Set, E., Gozpinar, A.: A study on Hermite-Hadamard type inequalities for $s$-convex functions via conformable fractional integrals. Stud. Univ. Babes-Bolyai, Math. 62(3), 309-323 (2017)

14. Khalil, R., Horani, M.A., Yousef, A., Sababheh, M.: A new definition of fractional derivative. J. Comput. Appl. Math. 264, 65-70 (2014)

15. Kirane, M., Torebek, B.T.: Hermite-Hadamard, Hermite-Hadamard-Fejér, Dragomir-Agarwal and Pachpatte type inequalities for convex functions via fractional integrals. arXiv:1701.00092

16. Sarikaya, M.Z., Ertugral, F.: On the generalized Hermite-Hadamard inequalities. An. Univ. Craiova, Ser. Mat. Inform. 47(1), 193-213 (2020)

17. Baloch, I.A., Chu, Y.M.: Petrovíc-type inequalities for harmonic $h$-convex functions. J. Funct. Spaces 2020, Article ID $3075390(2020)$

18. Han, J., Mohammed, P.O., Zeng, H.: Generalized fractional integral inequalities of Hermite-Hadamard-type for a convex function. Open Math. 18, 794-806 (2020)

19. Kashuri, A., Liko, R.: Some new Hermite-Hadamard type inequalities and their applications. Studia Sci. Math. Hung. 56(1), 103-142 (2019)

20. Kashuri, A., Iqbal, S., Butt, S.I., Nasir, J., Nisar, K.S., Abdeljawad, T.: Trapezium-type inequalities for k-fractional integral via new exponential-type convexity and their applications. J. Math. 2020, Article ID 8672710 (2020) 
21. Mohammed, P.O., Abdeljawad, T., Zeng, S., Kashuri, A.: Fractional Hermite-Hadamard integral inequalities for a new class of convex functions. Symmetry 12, 1485 (2020)

22. Baleanu, D., Kashuri, A., Mohammed, P.O., Meftah, B.: General Raina fractional integral inequalities on coordinates of convex functions. Adv. Differ. Equ. 2021, 82 (2021)

23. Sroysang, B.: Generalizations on some Hermite-Hadamard type inequalities for differential convex function with applications to weighted means. Sci. World J. 2014, Article ID 717164 (2014)

24. Xi, B.Y., Qi, F.: Some integral inequalities of Hermite-Hadamard type for convex functions with applications to means. J. Funct. Spaces Appl. 2012, Article ID 980438 (2012)

25. Akkurt, A., Sarikaya, M.Z., Budak, H., Yildirim, H.: On the Hadamard's type inequalities for co-ordinated convex functions via fractional integrals. J. King Saud Univ., Sci. 29, 380-387 (2017)

26. Cesarano, C., Pierpaolo, N., Paolo, E.R.: Pseudo-Lucas functions of fractional degree and applications. Axioms 10, 51 (2021)

27. Kober, H.: On fractional integrals and derivatives. Q. J. Math. 11(1), 193-211 (1940)

28. Noor, M.A., Awan, M.U., Noor, K.I., Postolache, M.: Some integral inequalities for $p$-convex functions. Filomat 30(9), 2435-2444 (2016)

29. Noor, M.A., Awan, M.U., Noor, K.I. Integral inequalities for two-dimensional pq-convex functions. Filomat 30(2), 343-351 (2016)

30. Raees, M., Anwar, M.: On Hermite-Hadamard type inequalities of coordinate $\left(r_{1}, \hbar_{1}\right)-\left(r_{2}, \hbar_{2}\right)$-convex function via Katugampola fractional integral. Filomat 33(15), 4785-4802 (2019)

31. Sarikaya, M.Z:: On the Hermite-Hadamard-type inequalities for coordinated convex function via fractional integrals. Integral Transforms Spec. Funct. 25(2), 134-147 (2014)

32. Sabatier, J.A.T.M.J., Agrawal, O.P., Machado, J.A.T.: Advances in Fractional Calculus. The Netherlands, vol. 4. Springer, Dordrecht (2007)

\section{Submit your manuscript to a SpringerOpen ${ }^{\circ}$ journal and benefit from:}

- Convenient online submission

- Rigorous peer review

- Open access: articles freely available online

- High visibility within the field

- Retaining the copyright to your article

Submit your next manuscript at $\boldsymbol{\nabla}$ springeropen.com 